\title{
Discretization of Multipole Sources in a Finite Difference Setting for Wave Propagation Problems
}

\author{
Mario J. Bencomo ${ }^{1}$ and William Symes ${ }^{2}$ \\ ${ }^{1}$ Institute for Computational and Experimental Research in Mathematics, \\ Brown University, \\ Providence, RI 02912 USA \\ ${ }^{2}$ The Rice Inversion Project, \\ Rice University, \\ Houston, TX 7ro05-1892 USA
}

(June 20, 2018)

Running head: Multipole Source Discretizations

\begin{abstract}
Seismic sources are commonly idealized as point-sources due to their small spatial extent relative to seismic wavelengths. The acoustic isotropic point-radiator is inadequate as a model of seismic wave generation for seismic sources that are known to exhibit directivity. Therefore, accurate modeling of seismic wavefields must include source representations generating anisotropic radiation patterns. Such seismic sources can be modeled as linear combinations of multipole point-sources. In this paper we present a method for discretizing multipole sources in a finite difference setting, an extension of the moment matching conditions developed for the Dirac delta function in other applications. We also provide the necessary analysis and numerical evidence to demonstrate the accuracy of our singular source ap-
\end{abstract}


proximations. In particular, we develop a weak convergence theory for the discretization of a family of symmetric hyperbolic systems of first-order partial differential equations, with singular source terms, solved via staggered-grid finite difference methods. Numerical experiments demonstrate a stronger result than what is presented in our convergence theory, namely, optimal convergence rates of numerical solutions are achieved point-wise in space away from the source if an appropriate source discretization is used. 


\section{INTRODUCTION}

Seismic sources are commonly idealized as concentrated at a source point due to their small spatial extent relative to seismic wavelengths. A simple and familiar example of a wave propagation model with spatially concentrated source is the isotropic point-radiator problem for the acoustic wave equation in Euclidean 3-space (Courant and Hilbert, 1962):

$$
\begin{aligned}
\frac{\partial^{2}}{\partial t^{2}} p(\mathbf{x}, t)-c^{2} \nabla^{2} p(\mathbf{x}, t) & =f(\mathbf{x}, t) \equiv w(t) \delta(\mathbf{x}), \\
p(\mathbf{x}, t) & =0, \quad t<<0
\end{aligned}
$$

$\delta(\mathbf{x})$ being the Dirac delta function. The solution $p$ is spherically symmetric:

$$
p(\mathbf{x}, t)=\frac{w\left(t-\frac{r}{c}\right)}{4 \pi c^{2} r}, \quad r=\sqrt{\mathbf{x}^{T} \mathbf{x}}
$$

Both active and earthquake seismic sources, however, generate spatially asymmetric wavefields (see for example Shearer (2009), Yilmaz (2001)). The acoustic isotropic point-radiator is therefore inadequate as a model of seismic wave generation and propagation because of its prediction of spatial symmetry. The symmetry of the solution arises in part from the spherical symmetry of the right-hand side in equation 1 . Therefore, accurate modeling of seismic wavefields must include energy source (right-hand side) representations generating anisotropic radiation patterns.

A multipole, or multipole source in the context of source modeling, is a finite linear combination of partial derivatives of the spatial Dirac delta function. Such sources combine localization of energy and anisotropic radiation pattern. In fact, Peetre's Theorem (Hörmander, 1969) implies that any function of space and time $f(\mathbf{x}, t)$ concentrated en- 
tirely at a point in space (of point support) is a multipole of finite order $N \geq 0$,

$$
f(\mathbf{x}, t)=\sum_{|\mathbf{s}|=0}^{N} w_{\mathbf{s}}(t) D^{\mathbf{s}} \delta\left(\mathbf{x}-\mathbf{x}^{*}\right)
$$

in which we have introduced multi-index notation: for spatial dimension $d=\{1,2,3\}$ and multi-index (integer d-tuple) $\mathbf{s}=\left(s_{1}, \ldots, s_{d}\right)$, the s-mixed partial derivative operator, denoted $D^{\mathbf{s}}$, and its (total) order $|\mathbf{s}|$, are defined as

$$
D^{\mathbf{s}}=\prod_{i=1}^{d}\left(\frac{\partial}{\partial x_{i}}\right)^{s_{i}}, \quad|\mathbf{s}|=\sum_{i=1}^{d} s_{i}
$$

The coefficient time functions $w_{\mathbf{s}}(t)$ may be scalar-, vector-, or tensor-valued, according to the nature of the quantity (pressure, velocity, or stress) in the equation in which $f(\mathbf{x}, t)$ appears as right-hand side. Multipoles may approximate arbitrary sources highly localized on the wavelength scale, in the sense of generating approximately the same field away from the source location, and for this reason have enjoyed widespread use in the representation of seismic sources (Shearer, 2009).

While finite element methods are also used in earthquake seismic modeling and inversion (Komatisch et al., 2000; Cohen, 2002; Epanomeritakis et al., 2009), this paper focuses on regular (rectangular) grid finite difference methods, in particular the staggered-grid variety, which are widely used for basin- and exploration-scale modeling; see Moczo et al. (2006) for an excellent overview and many older references. Such methods pose an immediate problem for singular source models such as multipoles: finite difference algorithms "know" only gridded fields, so a source located at an arbitrary point $\mathrm{x}^{*}$ in space must be represented somehow by virtual sources at nearby grid points. This task is complicated by the nature 
of the field, as is evident for instance from inspection of the 3 -D analytical function 2 : solutions of the acoustic equations with singular right-hand sides are generally themselves singular at the source point, so the Taylor-series based analysis of finite difference accuracy does not apply.

The focus of this paper is to study singular source discretizations, particularly in the context of staggered-grid finite difference solvers for a family of wave propagation differential equations (e.g., linear acoustics and elasticity in first-order form) with multipole sources. Specifically, we are interested in the following class of symmetric hyperbolic systems of first-order partial differential equations: find vector-valued fields $(u, v)$ such that

$$
\begin{aligned}
A \frac{\partial}{\partial t} u+P^{T} v & =f \\
B \frac{\partial}{\partial t} v-P u & =g
\end{aligned}
$$

for given source terms $(f, g)$. Operators $A$ and $B$ are bounded symmetric matrix-valued and represent medium parameters. $P$ is a first-order spatial differential operator with constant coefficients, e.g., $P$ is the gradient in the acoustic equations 23 with $P^{T}$ being the negative of the divergence. We directly discretize multipole sources using discrete moment conditions as developed by Waldén (1999), and elaborated by Tornberg and Engquist (2004) for multi-dimensional approximations. Let $\left(u_{h}, v_{h}\right)$ denote the finite difference solution to 5 via staggered-grid finite differences, with discretized source terms $\left(f_{h}, g_{h}\right)$, where $h>0$ denotes the grid size. The class of staggered-grid finite difference methods we deal with are second-order in time and $2 p$-order in space accurate, and we refer to such a scheme as being of order $2-2 p$.

Our main contribution is a weak convergence theory, a novel approach to the singular 
source approximation problem, that is applicable to 5 in that $\left(u_{h}, v_{h}\right)$ converge to $(u, v)$ given that source approximations $\left(f_{h}, g_{h}\right)$ converge to $(f, g)$ as $h \rightarrow 0$, both in a weak sense which we make clear in the theory section. In particular, we show

$$
\langle(u, v),(\tilde{f}, \tilde{g})\rangle-\left\langle\left(u_{h}, v_{h}\right),(\tilde{f}, \tilde{g})\right\rangle=O\left(h^{q}+\Delta t^{2}+\left(h^{2 p}+\Delta t\right) h^{-N^{*}-d / 2}\right)
$$

given smooth test functions $(\tilde{f}, \tilde{g})$. Inner products $\langle\cdot, \cdot\rangle$ are interpreted as standard $L^{2}$ space-time inner products in a continuum or discrete sense depending on their arguments. Estimate 6 thus depends on singular source approximation order $q$, spatial half-order $p$ for a staggered-grid finite difference scheme of order $2-2 p$, the maximum multipole order between $f$ and $g$ denoted by $N^{*}$, and spatial dimension $d$. Numerical results presented here, consistent with other similar works (e.g., Petersson and Sjögreen (2010)), appear to indicate, however, stronger convergence results: optimal convergence point-wise away from source. In particular, we report second and fourth order rates when studying the spatial convergence of 2-2 and 2-4 finite difference methods respectively, where the source approximation order matched the spatial order of the numerical scheme.

The analysis and numerical examples presented by Waldén (1999), though limited to the 1-D Helmholtz equation, demonstrated point-wise convergence of numerical solutions with optimal convergence rates (as suggested by the numerical scheme) away from the source location when appropriately discretizing the singular source term. The theory of singular source approximations has been further extended to a range of applications, most notably for the Dirac delta function in the context of the immersed boundary method (Peskin, 2002). Several authors have addressed questions regarding the convergence of source approximations and subsequently their effect on solutions to more complicated differential equations. 
Consider the following abstract problem: find $u$ such that

$$
\mathcal{L} u=f,
$$

for some differential operator $\mathcal{L}$ and singular source term $f$. Define the regularization of problem 7 by replacing $f$ with some regular (at least piecewise continuous) function $f^{\epsilon}$ parameterized by regularization parameter $\epsilon>0$, that is, find $u^{\epsilon}$ such that

$$
\mathcal{L} u^{\epsilon}=f^{\epsilon}
$$

Regularized source term $f^{\epsilon}$ is said to approximate $f$ in that $f^{\epsilon} \rightarrow f$ as $\epsilon \rightarrow 0$ in some sense. The end goal is of course to have $u^{\epsilon}$ approximate true solution $u$, i.e.,

$$
\lim _{\epsilon \rightarrow 0}\left\|u^{\epsilon}-u\right\|_{X}=0
$$

under some suitable norm $\|\cdot\|_{X}$.

Tornberg and Engquist (2003) have studied the regularization error $\left\|u^{\epsilon}-u\right\|_{X}$, pointwise away from the source location. Their analysis is based on a simple ODE case where they prove convergence of regularized solutions $u^{\epsilon}$ if $f^{\epsilon}$ satisfy what we call the continuum moment conditions. We use the qualifier "continuum" to differentiate at times between the discrete moment conditions. Recent work by Hosseini et al. (2016) addresses the mode of convergence of $f^{\epsilon} \rightarrow f$ subject to regularized source terms satisfying the continuum moment conditions, mainly convergence in a weak-* topology (distribution sense) and in a weighted Sobelev norm. Both Tornberg and Engquist (2003) and Hosseini et al. (2016) argue that $f^{\epsilon} \rightarrow f$ implies $u^{\epsilon} \rightarrow u$ as $\epsilon \rightarrow 0$, point-wise away from the source location, 
in particular for elliptic operators $\mathcal{L}$. This argument hinges on the integral representation of elliptic operators and the smoothness of their kernel (i.e., Green's functions) away from source location.

Suppose that the regularized problem 8 is discretized with mesh or cell size $h>0$;

$$
\mathcal{L}_{h} u_{h}^{\epsilon}=f_{h}^{\epsilon}
$$

In the context of finite difference methods $\mathcal{L}_{h}$ is the finite difference approximation of differential operator $\mathcal{L}$. The discrete source term $f_{h}^{\epsilon}$ can be interpreted as the discretization (e.g., sampling over grid points) of the regularized source term $f^{\epsilon}$ generated by the continuum moment conditions, or as the direct discretization of $f$ through the discrete moment conditions. In practice the regularization parameter $\epsilon$ is related to the discretization parameter $h$, that is, $\epsilon=\epsilon(h)$ such that

$$
\lim _{h \rightarrow 0} \epsilon(h)=0 .
$$

Tornberg and Engquist (2004) provided insight into the convergence of $f_{h}^{\epsilon}$ as $h \rightarrow 0$ for direct discretizations of $f$ via the discrete moment conditions, in particular for discretizations whose support is proportional to $\epsilon$ and $\epsilon(h)=O(h)$. Consistent with results by Waldén (1999), Tornberg and Engquist (2004) demonstrated the convergence of numerical solutions $u_{h}^{\epsilon}$ for problem 9 , in particular

$$
\left\|u_{h}^{\epsilon}-u\right\|_{X}=O\left(h^{p}\right)
$$

where $p$ is the convergence rate of the numerical scheme and $f_{h}^{\epsilon}$ satisfies a sufficient number of moment conditions. The norm $\|\cdot\|_{X}$ in this case coincides with the sup-norm with a deleted neighborhood containing the source location. Theory presented by Tornberg and 
Engquist (2004) is based on analysis of the 1-D Poisson equation discretized by second- and fourth-order finite difference approximations.

Green's functions for hyperbolic problems are singular at the wavefront they propagate as well as at the source location, thus the analysis of Tornberg and Engquist (2004) does not apply. Recent work by Petersson et al. (2016) presented some of the most relevant analysis based on centered difference approximations to the 1-D advection equation with singular source term. They show that the discrete moment conditions are necessary but not sufficient for the convergence of numerical solutions at optimal rates away from the source location. They demonstrated that smoothness conditions are also required to achieve convergence; these conditions are based on Fourier analysis and the ability of the finite difference operators at play to resolve spurious modes injected by the singular source approximation.

The remainder of the paper is organized as follows: In the theory section we begin by presenting an overview of the singular source approximation method via continuum and discrete moment conditions. We focus primarily on source approximations of narrow support, as discussed in Tornberg and Engquist (2004), though we provide explicit formulas for approximations of arbitrary order. Moreover, we show that the discrete moment conditions in fact define a sequence of continuum functions that converge to target distributions in a weak sense, a new result. The theory section also covers known convergence results of staggered-grid finite difference methods via energy estimates, applied to our set of differential equations under smooth coefficients and smooth source terms. At this point we present our weak convergence theory. The last section covers numerical results, mainly for staggered-grid finite difference solutions to the 2-D acoustic equations in first-order form 
with multipole sources. Consistent with numerical results presented in the literature (see for example Petersson and Sjögreen (2010)), we observe optimal convergence rates of our numerical solutions when the source discretization satisfied the proper number of moment conditions. 


\title{
THEORY
}

\section{Singular Source Approximation}

\author{
(Continuum) Moment Conditions
}

We begin by noting that the delta function and its derivatives (and thus multipoles) are not actually functions but rather so-called distributions, functionals that return a real number when applied to a test function. Let $\mathcal{D}$ denote the space of test functions over $\mathbb{R}^{d}$, that is, the space of $C_{0}^{\infty}\left(\mathbb{R}^{d}\right)$ endowed with the standard topology of test functions. The set of distributions is given by the dual of the space of test functions, denoted by $\mathcal{D}^{\prime}$. It is conventional to represent the application of a distribution on a function by the integral of the product, even when the distribution is not actually a function that can be integrated in the usual sense. For example, given multi-index $\mathbf{s}=\left(s_{1}, \ldots, s_{d}\right)$, the $\mathbf{s}$-mixed partial derivative of the Dirac delta function, shifted by $\mathbf{x}^{*} \in \mathbb{R}^{d}$, is defined by

$$
\int_{\mathbb{R}^{d}} D^{\mathbf{s}} \delta\left(\mathbf{x}-\mathbf{x}^{*}\right) \psi(\mathbf{x}) d \mathbf{x}=(-1)^{|\mathbf{s}|} D^{\mathbf{s}} \psi\left(\mathbf{x}^{*}\right), \quad \forall \psi \in \mathcal{D}
$$

The key idea for constructing approximations to $D^{\mathbf{s}} \delta\left(\mathbf{x}-\mathbf{x}^{*}\right)$ is based on mimicking the behavior of the target distribution on polynomials, reminiscent of finite difference approximations for differential operators. Consider $\psi(\mathbf{x})=\left(\mathbf{x}-\mathbf{x}^{*}\right)^{\alpha}$, with multi-index $\alpha=\left(\alpha_{1}, \ldots, \alpha_{d}\right)$, where multi-indexed monomials are interpreted as the product of monomials in each dimension,

$$
\mathbf{x}^{\alpha}=\prod_{k=1}^{d} x_{k}^{\alpha_{k}}
$$


It can be shown that

$$
\int_{\mathbb{R}^{d}} D^{\mathbf{s}} \delta\left(\mathbf{x}-\mathbf{x}^{*}\right) \psi(\mathbf{x}) d \mathbf{x}=\mathbf{s} !(-1)^{|\mathbf{s}|} \delta_{\mathbf{s} \alpha}
$$

where $\delta_{\mathbf{s} \alpha}$ is the Kronecker delta, defined as follows for multi-indexes,

$$
\delta_{\mathbf{s} \alpha}:=\prod_{k=1}^{d} \delta_{s_{k} \alpha_{k}}
$$

Given $\eta \in L_{0}^{1}\left(\mathbb{R}^{d}\right)$ (i.e., integrable function of compact support) and multi-index $\alpha$, the $\alpha$-moment of $\eta$ centered at $\mathbf{x}^{*} \in \mathbb{R}^{d}$, denoted by $M^{\alpha}\left(\cdot, \mathbf{x}^{*}\right)$, is defined as

$$
M^{\alpha}\left(\eta, \mathbf{x}^{*}\right):=\int_{\mathbb{R}^{d}} \eta(\mathbf{x})\left(\mathbf{x}-\mathbf{x}^{*}\right)^{\alpha} d \mathbf{x}
$$

For given nonnegative integer $q$ and multi-index $\mathbf{s}$, the function $\eta$ is said to satisfy the continuum $(q, \mathbf{s})$-moment conditions at $\mathbf{x}^{*} \in \mathbb{R}^{d}$ if

$$
M^{\alpha}\left(\eta, \mathbf{x}^{*}\right)=\mathbf{s} !(-1)^{|\mathbf{s}|} \delta_{\mathbf{s} \alpha}, \quad \forall|\alpha|=0, \ldots, q+|\mathbf{s}|-1
$$

If $\eta$ satisfies the $(q, \mathbf{s})$-moment conditions at $\mathbf{x}^{*}$, then its associated distribution, that is

$$
\int_{\mathbb{R}^{d}} \eta(\mathbf{x}) \psi(\mathbf{x}) d \mathbf{x}, \quad \forall \psi \in \mathcal{D}
$$

is an approximation to $D^{\mathbf{s}} \delta\left(\mathbf{x}-\mathbf{x}^{*}\right)$ in that it is exact on polynomials of order $q+|\mathbf{s}|-1$. The following theorem states that a sequence of (regular) distributions of compact support, satisfying the $(q, \mathbf{s})$-moment conditions, will converge in the weak-* topology at a rate $q$ to the target distribution as the width of the supports approach zero. Let $B\left(\mathbf{x}^{*}, \epsilon\right)$ denote the 
$d$-dimensional ball of radius $\epsilon$ centered at $\mathbf{x}^{*}$.

Theorem 1. Let nonnegative integer $q$, multi-index $\mathbf{s}$, and $\mathbf{x}^{*} \in \mathbb{R}^{d}$ be given. Suppose $\left\{\eta^{\epsilon}\right\} \subset L_{0}^{1}\left(\mathbb{R}^{d}\right)$ is a sequence of functions as $\epsilon \rightarrow 0$, where supp $\left(\eta^{\epsilon}\right) \subset B\left(\mathbf{x}^{*}, \epsilon\right)$. Furthermore, suppose that there exists a constant $K>0$ independent of $\epsilon$ such that

$$
\int_{\mathbb{R}^{d}}\left|\eta^{\epsilon}(\mathbf{x})\right|\left|\left(\mathbf{x}-\mathbf{x}^{*}\right)^{\alpha}\right| d \mathbf{x} \leq K, \quad \forall|\alpha|=|\mathbf{s}|
$$

If $\left\{\eta^{\epsilon}\right\}$ satisfy the $(q, \mathbf{s})$-moment conditions at $\mathbf{x}^{*}$, equation 11 , then the sequence of distribution they generate converges to $D^{\mathbf{s}} \delta\left(\mathbf{x}-\mathbf{x}^{*}\right)$ in the weak-* topology as $\epsilon \rightarrow 0$. In particular, if $\psi$ is of class $C^{q+|\mathbf{s}|}$ over $B\left(\mathbf{x}^{*}, \epsilon\right)$, then

$$
E:=\left|\int_{\mathbb{R}^{d}} D^{\mathbf{s}} \delta\left(\mathbf{x}-\mathbf{x}^{*}\right) \psi(\mathbf{x}) d \mathbf{x}-\int_{\mathbb{R}^{d}} \eta^{\epsilon}(\mathbf{x}) \psi(\mathbf{x}) d \mathbf{x}\right|=O\left(\epsilon^{q}\right)
$$

Proof. We first apply multi-variate Taylor's theorem to $\psi$, centered at $\mathbf{x}^{*}$ and truncated after $N=q+|\mathbf{s}|-1$ terms (Königsberger, 2013), assuming $\psi$ is $C^{q+|\mathbf{s}|}$ over $B\left(\mathbf{x}^{*}, \epsilon\right)$,

$$
\begin{aligned}
\int_{\mathbb{R}^{d}} \eta^{\epsilon}(\mathbf{x}) \psi(\mathbf{x}) d \mathbf{x} & =\int_{\mathbb{R}^{d}} \eta^{\epsilon}(\mathbf{x})\left(\sum_{|\alpha|=0}^{N} \frac{D^{\alpha} \psi\left(\mathbf{x}^{*}\right)}{\alpha !}\left(\mathbf{x}-\mathbf{x}^{*}\right)^{\alpha}+\sum_{|\beta|=N+1} R_{\beta}(\mathbf{x})\left(\mathbf{x}-\mathbf{x}^{*}\right)^{\beta}\right) d \mathbf{x} \\
& =\sum_{|\alpha|=0}^{N} \frac{D^{\alpha} \psi\left(\mathbf{x}^{*}\right)}{\alpha !}\left(\int_{\mathbb{R}^{d}} \eta^{\epsilon}(\mathbf{x})\left(\mathbf{x}-\mathbf{x}^{*}\right)^{\alpha} d \mathbf{x}\right)+\sum_{|\beta|=N+1} \int_{\mathbb{R}^{d}} \eta^{\epsilon}(\mathbf{x}) R_{\beta}(\mathbf{x})\left(\mathbf{x}-\mathbf{x}^{*}\right)^{\beta} d \mathbf{x}
\end{aligned}
$$

where $R_{\beta}$ is the remainder term,

$$
R_{\beta}(\mathbf{x})=\frac{|\beta|}{\beta !} \int_{0}^{1}(1-t)^{|\beta|-1} D^{\beta} \psi\left(\mathbf{x}^{*}+t\left(\mathbf{x}-\mathbf{x}^{*}\right)\right) d t
$$

Note that the term in the parenthesis in the bottom equation corresponds to the $\alpha$-moment 
centered at $\mathbf{x}^{*}$ with $|\alpha| \leq q+|\mathbf{s}|-1$, hence the $(q, \mathbf{s})$-moment conditions apply;

$$
\begin{aligned}
\int_{\mathbb{R}^{d}} \eta^{\epsilon}(\mathbf{x}) \psi(\mathbf{x}) d \mathbf{x} & =\sum_{|\alpha|=0}^{N} \frac{1}{\alpha !} D^{\alpha} \psi\left(\mathbf{x}^{*}\right)\left(\mathbf{s} !(-1)^{|\mathbf{s}|} \delta_{\mathbf{s} \alpha}\right)+\sum_{|\beta|=N+1} \int_{\mathbb{R}^{d}} \eta^{\epsilon}(\mathbf{x}) R_{\beta}(\mathbf{x})\left(\mathbf{x}-\mathbf{x}^{*}\right)^{\beta} d \mathbf{x} \\
& =(-1)^{|\mathbf{s}|} D^{\mathbf{s}} \psi\left(\mathbf{x}^{*}\right)+\sum_{|\beta|=N+1} \int_{\mathbb{R}^{d}} \eta^{\epsilon}(\mathbf{x}) R_{\beta}(\mathbf{x})\left(\mathbf{x}-\mathbf{x}^{*}\right)^{\beta} d \mathbf{x} .
\end{aligned}
$$

The remainder term is bounded uniformly over $B\left(\mathbf{x}^{*}, \epsilon\right)$,

$$
\sup _{\mathbf{x} \in B\left(\mathbf{x}^{*}, \epsilon\right)}\left|R_{\beta}(\mathbf{x})\right| \leq C(\beta, \psi):=\frac{1}{\beta !} \max _{|\alpha|=|\beta|} \max _{\mathbf{y} \in B\left(\mathbf{x}^{*}, \epsilon\right)}\left|D^{\alpha} \psi(\mathbf{y})\right|
$$

using the fact that $\psi \in C^{N+1}$ in $B\left(\mathbf{x}^{*}, \epsilon\right)$. This gives the following error estimate,

$$
E \leq \sum_{|\beta|=N+1} C(\beta, \psi) \int_{B\left(\mathbf{x}^{*}, \epsilon\right)}\left|\eta^{\epsilon}(\mathbf{x})\right|\left|\left(\mathbf{x}-\mathbf{x}^{*}\right)^{\beta}\right| d \mathbf{x} .
$$

Let $\gamma$ be a multi-index such that $|\gamma|=q$, thus $|\beta-\gamma|=|\mathbf{s}|$. This yields,

$$
\begin{aligned}
E & \leq \sum_{|\beta|=N+1} C(\beta, \psi)\left(\sup _{\mathbf{x} \in B\left(\mathbf{x}^{*}, \epsilon\right)}\left|\left(\mathbf{x}-\mathbf{x}^{*}\right)^{\gamma}\right|\right) \int_{B\left(\mathbf{x}^{*}, \epsilon\right)}\left|\eta^{\epsilon}(\mathbf{x})\right|\left|\left(\mathbf{x}-\mathbf{x}^{*}\right)^{\beta-\gamma}\right| d \mathbf{x} \\
& \leq \sum_{|\beta|=N+1} C(\beta, \psi)\left(\sup _{\mathbf{x} \in B\left(\mathbf{x}^{*}, \epsilon\right)}\left|\left(\mathbf{x}-\mathbf{x}^{*}\right)^{\gamma}\right|\right) K \\
& =O\left(\epsilon^{q}\right)
\end{aligned}
$$

Theorem 1 and moment conditions given in equation 11 are extensions of what is presented in Hosseini et al. (2016) for $|\mathbf{s}| \neq 0$. Given equation 13, we refer to $q$ as the singular source approximation order and $\eta^{\epsilon}$ as being a $q$-order approximation of $D^{\mathbf{s}} \delta\left(\mathbf{x}-\mathbf{x}^{*}\right)$. 


\section{Discrete Moment Conditions}

We define the regular grid centered at $\mathbf{x}_{0} \in \mathbb{R}^{d}$ with cell size $\mathbf{h}=\left(h_{1}, \ldots, h_{d}\right)>\mathbf{0}$, as the collection of points denoted by $\mathcal{G}\left(\mathbf{x}_{0}, \mathbf{h}\right)$,

$$
\mathcal{G}\left(\mathbf{x}_{0}, \mathbf{h}\right)=\left\{\mathbf{x}_{n}=\left(x_{1, n_{1}}, \ldots, x_{d, n_{d}}\right), \mathbf{n} \in \mathbb{Z}^{d}\right\}
$$

where

$$
x_{k, n_{k}}=x_{0, k}+h_{k} n_{k}, \quad \forall k=1, \ldots, d .
$$

Note that there is no reason to assume that the grid cell is cubical, it may have different lengths along different axes. However, we assume that the grid is refined by scaling a characteristic grid cell size $h$ (e.g., $h=\max _{k} h_{k}$ ) and which we will use to denote grid functions and other grid-dependent quantities with subscript $h$ even in dimensions higher than one.

The obvious definition of the discrete $\alpha$-moment, centered at $\mathbf{x}^{*} \in \mathbb{R}^{d}$ (note that $\mathbf{x}^{*}$ need not coincide with a grid point in $\mathcal{G}\left(\mathbf{x}_{0}, \mathbf{h}\right)$ ), of a grid function $\eta_{h}: \mathcal{G}\left(\mathbf{x}_{0}, \mathbf{h}\right) \rightarrow \mathbb{R}$ is given as follows:

$$
M_{h}^{\alpha}\left(\eta_{h}, \mathbf{x}^{*}\right):=\left(\prod_{k=1}^{d} h_{k}\right) \sum_{\mathbf{x} \in \mathcal{G}\left(\mathbf{x}_{0}, \mathbf{h}\right)} \eta_{h}(\mathbf{x})\left(\mathbf{x}-\mathbf{x}^{*}\right)^{\alpha} .
$$

It is worth pointing out that the discrete moment defined above is dependent on choice of grid, in particular dependent on the source location $\mathbf{x}^{*}$ relative to the grid. Similar to the continuum moment conditions (equation 11), grid function $\eta_{h}$ is said to satisfy the discrete 
$(q, \mathbf{s})$-moment conditions at $\mathbf{x}^{*} \in \mathbb{R}^{d}$ if

$$
M_{h}^{\alpha}\left(\eta_{h}, \mathbf{x}^{*}\right)=\mathbf{s} !(-1)^{|\mathbf{s}|} \delta_{\mathbf{s} \alpha}, \quad \forall|\alpha|=0, \ldots, q+|\mathbf{s}|-1
$$

The following theorem is a discrete analogue of theorem 1.

Theorem 2. Let nonnegative integer $q$, multi-index $\mathbf{s}$, and $\mathbf{x}^{*} \in \mathbb{R}^{d}$ be given. Suppose $\left\{\eta_{h}^{\epsilon}\right\}$ is a sequence of grid functions $\eta_{h}^{\epsilon}: \mathcal{G}\left(\mathbf{x}_{0}, \mathbf{h}\right) \rightarrow \mathbb{R}$ as $\epsilon \rightarrow 0$. Furthermore, assume that the support of $\eta_{h}^{\epsilon}$ is contained in $B\left(\mathbf{x}^{*}, \epsilon\right)$ with $\epsilon=O(h)$, and that there exists constant $K>0$ independent of $\epsilon$ such that

$$
\left(\prod_{k=1}^{d} h_{k}\right) \sum_{\mathbf{x} \in \mathcal{G}\left(\mathbf{x}_{0}, \mathbf{h}\right)}\left|\eta_{h}^{\epsilon}(\mathbf{x})\right|\left|\left(\mathbf{x}-\mathbf{x}^{*}\right)^{\alpha}\right| \leq K, \quad \forall|\alpha|=|\mathbf{s}| .
$$

If $\left\{\eta_{h}^{\epsilon}\right\}$ satisfy the discrete $(q, \mathbf{s})$-moment conditions at $\mathbf{x}^{*}$ (equation 14) and $\psi$ is of class $C^{q+|\mathbf{s}|}$ over $B\left(\mathbf{x}^{*}, \epsilon\right)$, then

$$
\left|\int_{\mathbb{R}^{d}} D^{\mathbf{s}} \delta\left(\mathbf{x}-\mathbf{x}^{*}\right) \psi(\mathbf{x}) d \mathbf{x}-\left(\prod_{k=1}^{d} h_{k}\right) \sum_{\mathbf{x} \in \mathcal{G}\left(\mathbf{x}_{0}, \mathbf{h}\right)} \eta_{h}^{\epsilon}(\mathbf{x}) \psi(\mathbf{x})\right|=O\left(h^{q}\right) .
$$

Proof. The proof of this theorem is omitted since it is nearly identical to that of the continuum case (theorem 1), replacing integrals with summations over grid points. The jump from $O\left(\epsilon^{q}\right)$ to $O\left(h^{q}\right)$ follows from $\epsilon=O(h)$.

Theorem 2 and discrete moment conditions 14 are generalizations of work by Tornberg and Engquist (2004) for $|\mathbf{s}| \neq 0$. The discrete moment conditions are also an extension of Waldén (1999) for dimensions higher than one. We now discuss with more detail how to construct said sequences of gridded and continuum functions starting in 1-D. 


\section{1-D Constructions}

We make the choice of having 1-D gridded approximations be centered at source location $x^{*} \in \mathbb{R}^{d}$, and define them to be zero outside the interval $\left[-\epsilon+x^{*}, \epsilon+x^{*}\right)$, with $2 \epsilon=N h$ for some positive integer $N$. In other words, there exists $N$ grid points, denoted by $\left\{\tilde{x}_{\ell}\right\}_{\ell=1}^{N}$, such that they are contained in the interval $\left[-\epsilon+x^{*}, \epsilon+x^{*}\right)$ for a given grid $\mathcal{G}\left(x_{0}, h\right)$. These

grid points $\left\{\tilde{x}_{\ell}\right\}_{\ell=1}^{N}$ are referred to as the stencil points of the approximation. We assume that the stencil points are ordered, i.e., $\tilde{x}_{1}<\tilde{x}_{2}<\cdots<\tilde{x}_{N}$. The discrete $(q, s)$-moment conditions thus results in a $N \times(q+s)$ system of equations for the grid function $\eta_{h}^{\epsilon}$ evaluated at stencil points,

$$
\mathbf{A d}=\mathbf{b}
$$

with

$$
\{\mathbf{A}\}_{k \ell}=\left(\tilde{x}_{\ell}-x^{*}\right)^{k-1}, \quad\{\mathbf{d}\}_{\ell}=\eta_{h}^{\epsilon}\left(\tilde{x}_{\ell}\right), \quad\{\mathbf{b}\}_{k}=\frac{s !(-1)^{s}}{h} \delta_{s, k-1}
$$

for $\ell=1, \ldots, N$ and $k=1, \ldots, q+s$. Note that $\mathbf{A}$ is a Vandermonde matrix of full rank and is guaranteed a solution if $N \geq q+s$ and no solution for $N<q+s$ under general $x^{*} \in \mathbb{R}$.

We choose the case where $N=q+s$, which we refer to as grid functions of narrow support. The system above will result in a unique solution for a given $x^{*} \in \mathbb{R}$. In fact the inverse matrix for $\mathbf{A}$ can be written explicitly using the following Vandermonde matrix 
inverse formula:

$$
\left\{\mathbf{A}^{-1}\right\}_{\ell k}=\left\{\begin{array}{cl}
(-1)^{N-k} \frac{\sum_{\substack{1 \leq m_{1}<\cdots<m_{N-k} \leq N \\
m_{1}, \ldots, m_{N-k} \neq \ell}}\left(\tilde{x}_{m_{1}}-x^{*}\right) \cdots\left(\tilde{x}_{m_{N-k}}-x^{*}\right)}{\prod_{\substack{1 \leq m \leq N \\
m \neq \ell}}\left(\tilde{x}_{\ell}-\tilde{x}_{m}\right)} & \text { for } 1 \leq k \leq N \\
\frac{1}{\prod_{\substack{1 \leq m \leq N \\
m \neq \ell}}\left(\tilde{x}_{\ell}-\tilde{x}_{m}\right)}, & \text { for } k=N .
\end{array}\right.
$$

Given the particular form of right-hand side vector $\mathbf{b}$, it follows that $\mathbf{d}$ is simply the scaled $(s+1)$-column of $\mathbf{A}^{-1}$, whence

$$
\eta_{h}^{\epsilon}\left(\tilde{x}_{\ell}\right)=\left\{\begin{array}{cc}
\sum_{s !(-1)^{N-1}}\left(\tilde{x}_{m_{1}}-x^{*}\right) \cdots\left(\tilde{x}_{m_{q-1}}-x^{*}\right) & \\
\frac{h^{N} \prod_{\substack{1 \leq m_{1}<\cdots<m_{q-1} \leq N \\
m_{1}, \ldots, m_{q-1} \neq \ell}}(\ell-m)}{m \neq \ell} & \text { for } q>1 \\
\frac{s !(-1)^{s}}{h^{N} \prod_{\substack{1 \leq m \leq N \\
m \neq \ell}}(\ell-m)}, & \text { for } q=1 .
\end{array}\right.
$$

Note that the equation above above is dependent on $x^{*}$. To be more precise, approximation $\eta_{h}^{\epsilon}$ is actually dependent on the relative position of $x^{*}$ with respect to the stencil points $\left\{\tilde{x}_{\ell}\right\}_{\ell=1}^{N}$, or equivalently dependent on $x_{0}$ and $h$. For example, shifting the source location by $h$ would result in the same grid function $\eta_{h}^{\epsilon}$ though shifted by a grid point. However, arbitrary shifts in $x^{*}$ yield approximations that may vary by more than a simple translation. Given that equation 15 is unique for a particular source location, we show that imposing the discrete moment conditions over all $x^{*} \in \mathbb{R}$ indeed defines a function over the reals. Moreover, we show that these continuum functions satisfy the continuum moment 
conditions and thus define a sequence of distributions that converge to $D^{s} \delta$ in the weak-* topology, a result previously not known.

\section{Connection between Continuum and Discrete Moment Conditions}

We first focus on the $x^{*}=0$ case and define our continuum approximation $\eta^{\epsilon}$ to be zero outside $[-\epsilon, \epsilon)$, with $2 \epsilon=N h$ for $N=q+s$. Furthermore, we define $\eta^{\epsilon}$ to be piecewise polynomial over $N$ invervals:

$$
\eta^{\epsilon}(x)=\left\{\begin{aligned}
P_{\ell}(x), & x \in\left[a_{\ell}, a_{\ell+1}\right), \text { for } \ell=1, \ldots, N \\
0, & \text { otherwise }
\end{aligned}\right.
$$

where $P_{\ell}$ is some polynomial over the considered interval, and $a_{\ell}=-\epsilon+(\ell-1) h$ for $\ell=1, \ldots, N+1$. Given the support of our approximation, and a regular grid $\mathcal{G}\left(x_{0}, h\right)$ it follows that there are $N$ grid points contained in the interval $[-\epsilon, \epsilon)$, again denoted by $\left\{\tilde{x}_{\ell}\right\}_{\ell=1}^{N}$. In fact,

$$
\tilde{x}_{\ell} \in\left[a_{\ell}, a_{\ell+1}\right), \quad \forall \ell=1, \ldots, N
$$

Let $\ell^{*}$ be the index such that $0 \in\left[a_{\ell^{*}}, a_{\ell^{*}+1}\right)$ and define $\zeta \in(0, h]$ by $\zeta=a_{\ell^{*}+1}-\tilde{x}_{\ell^{*}}$. Thus, if we vary $x^{*}$ within the interval $(-\zeta, h-\zeta]$ if follows that

$$
\tilde{x}_{\ell}-x^{*} \in\left[a_{\ell}, a_{\ell+1}\right), \quad \ell=1, \ldots, N .
$$

Let $\eta_{h}^{\epsilon}\left(\cdot ; x^{*}\right)$ denote the grid function that satisfies the discrete $(q, s)$-moment conditions for a given $x^{*} \in(-\zeta, h-\zeta]$. Then $\eta^{\epsilon}\left(\tilde{x}_{\ell}-x^{*}\right):=\eta_{h}^{\epsilon}\left(\tilde{x}_{\ell} ; x^{*}\right)$ defines $\eta^{\epsilon}$ over $\left[a_{\ell}, a_{\ell+1}\right)$ by allowing $x^{*}$ to vary over the prescribed interval. Moreover, slightly modifying equation 15 
as a function of $x=\tilde{x}_{\ell}-x^{*}$ defines the $P_{\ell}(x)$ polynomials of $\eta^{\epsilon}$,

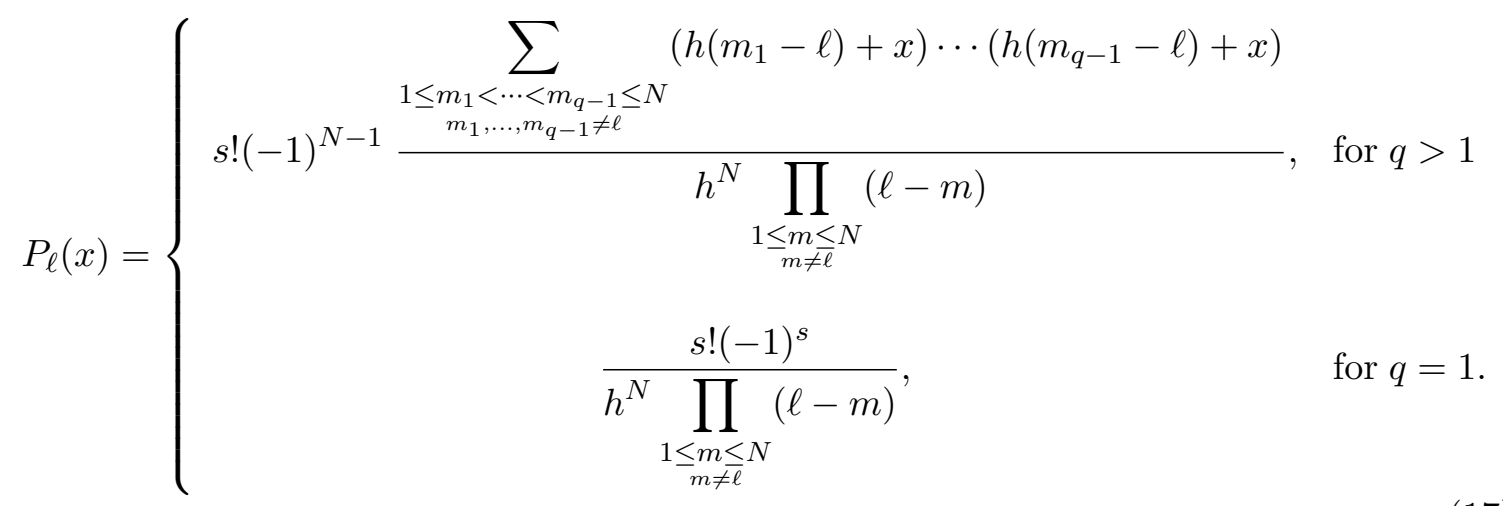

Inspection of equation 17 reveals that $P_{\ell}$ is a polynomial of degree $q-1$.

Theorem 3. Let nonnegative integer $q$, positive integer $s$, and $x^{*} \in \mathbb{R}$ be given. Suppose $\eta^{\epsilon}$ is constructed according to equations 16 and 17 for a given $h>0$. Then it follows that $\eta^{\epsilon}(x)$ is a q-order approximation of $D^{s} \delta(x)$.

Proof. In order to apply theorem 1 we need to verify that $\eta^{\epsilon}$ indeed satisfies the (continuum) $(q, s)$-moment conditions at 0 as well as the estimate given by equation 12 . We first evaluate the $\alpha$-moment of $\eta^{\epsilon}$ for $\alpha=0, \ldots, q+s-1$;

$$
\begin{aligned}
M^{\alpha}\left(\eta^{\epsilon}, 0\right) & =\int_{\mathbb{R}} \eta^{\epsilon}(x) x^{\alpha} d x \\
& =\sum_{\ell=1}^{N} \int_{a_{\ell}}^{a_{\ell+1}} P_{\ell}(x) x^{\alpha} d x .
\end{aligned}
$$

Applying the following change of variables, $x=a_{\ell}+\xi$ with $\xi \in[0, h)$, over each interval 
yields

$$
\begin{aligned}
M^{\alpha}\left(\eta^{\epsilon}, 0\right) & =\sum_{\ell=1}^{N} \int_{0}^{h} P_{\ell}\left(a_{\ell}+\xi\right)\left(a_{\ell}+\xi\right)^{\alpha} d \xi \\
& =\int_{0}^{h} \frac{1}{h}\left[h \sum_{\ell=1}^{N} P_{\ell}\left(a_{\ell}+\xi\right)\left(a_{\ell}+\xi\right)^{\alpha}\right] d \xi
\end{aligned}
$$

Note that the term in the bracket coincides with the discrete $\alpha$-moment of $\eta^{\epsilon}$ with respect to a uniform grid $\mathcal{G}\left(a_{1}, h\right)$ (containing stencil points $\left\{a_{\ell}\right\}_{\ell=1}^{N+1}$ ) for a source located at $x^{*}=-\xi$. In other words,

$$
h \sum_{\ell=1}^{N} P_{\ell}\left(a_{\ell}+\xi\right)\left(a_{\ell}+\xi\right)^{\alpha}=M_{h}^{\alpha}\left(\eta_{h}^{\epsilon},-\xi\right)
$$

where $\eta_{h}^{\epsilon}: \mathcal{G}\left(a_{1}, h\right) \rightarrow \mathbb{R}$ defined by $\eta_{h}^{\epsilon}(x)=\eta^{\epsilon}(x+\xi)$ satisfies the discrete $(q, s)$-moment conditions at $-\xi$ by construction. We can conclude

$$
\begin{aligned}
M^{\alpha}\left(\eta^{\epsilon}, 0\right) & =\int_{0}^{h} \frac{1}{h}\left[s !(-1)^{s} \delta_{s \alpha}\right] d \xi \\
& =s !(-1)^{s} \delta_{s \alpha} .
\end{aligned}
$$

Lastly, since $\eta^{\epsilon}$ consist of piecewise polynomials of order $q-1$ divided by a factor of $h^{N}$, where $N=q+s$, and $\epsilon=O(h)$, we have that

$$
\sup _{x \in B(0, \epsilon)}\left|\eta^{\epsilon}(x)\right|=O\left(\epsilon^{-s-1}\right) .
$$

Thus

$$
\int_{B(0, \epsilon)} d x\left|\eta^{\epsilon}(x)\right|\left|x^{s}\right| \leq \sup _{B(0, \epsilon)}\left|\eta^{\epsilon}(x)\right| \int_{B(0, \epsilon)} d x\left|x^{s}\right|=O(1)
$$

as required for estimate 12 
Constructions in Higher Dimensions via Tensor Products

We construct approximations in general $d$-dimension by taking tensor products of 1 -D approximations, similar to work by Tornberg and Engquist (2004) for $\mathbf{s}=\mathbf{0}$. Namely, given approximation order $q$ and multi-index $\mathbf{s}$, multivariate continuum approximation $\eta: \mathbb{R}^{d} \rightarrow \mathbb{R}$ is given by

$$
\eta(\mathbf{x})=\prod_{k=1}^{d} \eta^{k}\left(x_{k}\right)
$$

where $\eta^{k}: \mathbb{R} \rightarrow \mathbb{R}$ is a continuum function over the $k$-th axis as given by equations 16 and 17 , satisfying the $\left(q, s_{k}\right)$-moment conditions for each $k=1, \ldots, d$. It follows that these tensor approximations are indeed approximations to multipoles in the higher spatial dimension.

Theorem 4. Let nonnegative integer $q$, multi-index $\mathbf{s}$, and $\mathbf{x}^{*} \in \mathbb{R}^{d}$ be given. Suppose $\eta$ : $\mathbb{R}^{d} \rightarrow \mathbb{R}$ is a multi-variate grid function given by the tensor product of 1-D approximations $\eta^{k}: \mathbb{R} \rightarrow \mathbb{R}$. If $\eta^{k}$ satisfy the discrete $\left(q, s_{k}\right)$-moment conditions at $x_{k}^{*}$ for each $k=1, \ldots, d$, then it follows that $\eta$ satisfies the discrete $(q, \mathbf{s})$-moment conditions at $\mathbf{x}^{*}$.

Proof. Suppose for each $k=1, \ldots, d$ that $\eta^{k}$ satisfies the $\left(q, s_{k}\right)$-moment conditions at $x_{k}^{*}$. Let $\alpha$ be some multi-index with $|\alpha| \leq q+|\mathbf{s}|-1$. Note that,

$$
M^{\alpha}\left(\eta, \mathbf{x}^{*}\right)=\prod_{k=1}^{d} M^{\alpha_{k}}\left(\eta^{k}, x_{k}^{*}\right)
$$

Clearly, if $\alpha_{k} \leq q+s_{k}-1$ for all $k=1, \ldots, d$, then the result follows from the supposition. Same applies for $\alpha=\mathbf{s}$. Suppose then, that there exists index $\ell$ such that $\alpha_{\ell}>q+s_{\ell}-1$, 
that is $a_{\ell}=q+s_{\ell}-1+i$ for some $i \in \mathbb{N}$. Thus,

$$
\begin{aligned}
& |\alpha|=\sum_{k \neq \ell} \alpha_{k}+\alpha_{\ell}=\sum_{k \neq \ell} \alpha_{k}+q+s_{\ell}-1+i \leq q+|\mathbf{s}|-1 \\
\Longrightarrow & \sum_{k \neq \ell} \alpha_{k}+i \leq \sum_{k \neq \ell} s_{k} .
\end{aligned}
$$

which implies that $\alpha_{k}<s_{k}$ for at least one $k \neq \ell$; for this particular $k$, it follows that

$$
M^{\alpha_{k}}\left(\eta^{k}, x_{k}^{*}\right)=s_{k} !(-1)^{s_{k}} \delta_{s_{k} \alpha_{k}}=0
$$

since it has been established that $\alpha_{k} \neq s_{k}$, i.e., the product over $k$ is zero if $\mathbf{s} \neq \alpha$.

\section{Examples}

Numerical convergence rate tests in the following section will employ the singular source approximation discussed here, replacing multipole terms with grid functions to be used in finite difference schemes. Equation 15 gives an explicit formula for such grid functions, depending on the source location $\mathbf{x}^{*}$, multi-index $\mathbf{s}$, approximation order $q$, and of course the underlying finite difference grid. Alternatively, equations 16 and 17 define the continuum form of the approximations, where grid functions are obtained by shifting and sampling over the grid. The following figures plot 1-D and 2-D continuum approximations $\eta^{\epsilon}$, in particular, we plot second- and fourth-order approximations of $D^{s} \delta(\mathbf{x})$ for $s=0,1,2$ in 1 -D and $\mathbf{s}=(0,0),(0,1),(0,2)$ in 2 -D, with $h=1$. Two dimensional approximations are constructed via tensor product of 1-D approximations as discussed. In particular, the $q=2$ approximation for the 1-D Dirac delta function $(s=0)$ is none other than the well known hat/triangular function of unit mass. 


\section{Convergence Theory of Wave Equation Solutions with Multipole Sources}

We prove weak convergence of finite difference solutions with discrete multipole sources satisfying the discrete moment conditions discussed above. Convergence theory for smooth solutions is in some sense standard. The easily available results (those based on Lax's Equivalence Theorem) pertain to $L^{2}$ convergence for smooth solutions and smooth source terms, which we begin with.

Theoretical results presented here pertain to the family of symmetric hyperbolic systems of first-order partial differential equations for vector-valued fields $(u, v)$, defined over a domain $\Omega \subset \mathbb{R}^{d}$ in $d=\{1,2,3\}$ dimension space and some interval in time, given by 5 which we restate with greater detail. Let $k_{1} \in \mathbb{N}$ and $k_{2} \in \mathbb{N}$ denote the dimension of vector-fields $u$ and $v$ respectively. The systems we are interested in are of the following form:

$$
\begin{aligned}
A(\mathbf{x}) \frac{\partial}{\partial t} u(\mathbf{x}, t)+P^{T} v(\mathbf{x}, t) & =f(\mathbf{x}, t) \\
B(\mathbf{x}) \frac{\partial}{\partial t} v(\mathbf{x}, t)-P u(\mathbf{x}, t) & =g(\mathbf{x}, t)
\end{aligned}
$$

where

- $u \in L_{l o c}^{2}\left(\mathbb{R}, \mathcal{H}_{1}\right)$ and $v \in L_{l o c}^{2}\left(\mathbb{R}, \mathcal{H}_{2}\right)$ with Hilbert spaces $\mathcal{H}_{1}, \mathcal{H}_{2}$

- "coefficient" operators $A$ and $B$ are $k_{1} \times k_{1}$ and $k_{2} \times k_{2}$ matrix-valued functions respectively; we assume they are symmetric for all $\mathbf{x} \in \Omega$ and uniformly-positive 
definite, i.e., there exists constants $0<A_{*} \leq A^{*}$ and $0<B_{*} \leq B^{*}$ such that

$$
\begin{aligned}
& A_{*} I \leq A(\mathbf{x}) \leq A^{*} I, \quad \forall \mathbf{x} \in \Omega, \\
& B_{*} I \leq B(\mathbf{x}) \leq B^{*} I, \quad \forall \mathbf{x} \in \Omega ;
\end{aligned}
$$

- $P: \mathcal{H}_{1} \rightarrow \mathcal{H}_{2}$ is a constant coefficient, first-order differential operator of the form

$$
P u=\sum_{j=1}^{d} P_{j} \frac{\partial u}{\partial x_{j}}, \quad P_{j} \in \mathbb{R}^{k_{2} \times k_{1}}
$$

- source terms $(f, g)$ are for the time being assumed to be smooth, i.e., $f \in L_{l o c}^{2}\left(\mathbb{R}, \mathcal{H}_{1}\right)$ and $g \in L_{l o c}^{2}\left(\mathbb{R}, \mathcal{H}_{2}\right)$;

- we assume solution $(u, v)$ and source terms $(f, g)$ are causal, i.e., for $t<0$

$$
u(\mathbf{x}, t)=f(\mathbf{x}, t)=0, \quad v(\mathbf{x}, t)=g(\mathbf{x}, t)=0 .
$$

See Blazek et al. (2013) for proof of existence of solutions, stability, and other mathematical properties for a larger class of partial (integro-)differential systems for wave modeling, applicable to system 21 .

As an example, consider the acoustic equations in first-order (pressure-velocity) form:

$$
\begin{aligned}
\frac{1}{\kappa(\mathbf{x})} \frac{\partial}{\partial t} p(\mathbf{x}, t)+\nabla \cdot \mathbf{v}(\mathbf{x}, t) & =f(\mathbf{x}, t) \\
\rho(\mathbf{x}) \frac{\partial}{\partial t} \mathbf{v}(\mathbf{x}, t)+\nabla p(\mathbf{x}, t) & =\mathbf{g}(\mathbf{x}, t)
\end{aligned}
$$

where $u=p$ is the scalar pressure field and $v=\mathbf{v} \in \mathbb{R}^{d}$ is the vector particle velocity field; we take $\mathcal{H}_{1}=L^{2}(\Omega)$ and $\mathcal{H}_{2}=L^{2}(\Omega)^{d}$. Coefficient operator $A(\mathbf{x})=1 / \kappa(\mathbf{x})$ and 
$B(\mathbf{x})=\rho(\mathbf{x}) I$, with $\kappa$ denoting bulk-modulus and $\rho$ density of the medium; here $I \in \mathbb{R}^{d \times d}$ is the identity matrix. Lastly, the differential operator $P$ coincides with the gradient and its adjoint with the negative of the divergence,

$$
P=\left[\begin{array}{r}
\frac{\partial}{\partial x_{1}} \\
\vdots \\
\frac{\partial}{\partial x_{d}}
\end{array}\right], \quad P^{T}=-\left[\frac{\partial}{\partial x_{1}}, \ldots, \frac{\partial}{\partial x_{d}}\right]
$$

The discretization of continuum problem 21 is given by the following abstract staggered grid dynamical system:

$$
\begin{aligned}
& A_{h}\left(u_{h}^{n+1}-u_{h}^{n}\right)+P_{h}(r)^{T} v_{h}^{n+1 / 2}=r h f_{h}^{n+1 / 2} \\
& B_{h}\left(v_{h}^{n+1 / 2}-v_{h}^{n-1 / 2}\right)-P_{h}(r) u_{h}^{n}=r h g_{h}^{n}
\end{aligned}
$$

where

- $u_{h}^{n} \in H_{1, h}$ and $v_{h}^{n+1 / 2} \in H_{2, h}$ for a family of Hilbert spaces $H_{1, h}, H_{2, h}$ ("space of spatial-grid functions"), with $h>0$ ("cell size"). Superscript indexes in $u_{h}^{n}$ and $v_{h}^{n+1 / 2}$ refer to the discretized time axis at times $n \Delta t$ and $(n+1 / 2) \Delta t$ respectively, with time step size $\Delta t=r h ; r$ will play the role of the CFL constant, crucial for stability of the discretization. Note that the fields $u_{h}$ and $v_{h}$ are staggered in time;

- bounded self-adjoint positive-definite operators (discretizations of $A$ and $B$ )

$$
A_{h}: H_{1, h} \rightarrow H_{1, h}, \quad B_{h}: H_{2, h} \rightarrow H_{2, h}
$$

with upper and lower bounds uniform in $h$ : there exists constants $0<A_{*} \leq A^{*}$ and 
$0<B_{*} \leq B^{*}$ such that

$$
\begin{gathered}
A_{*}\left\|u_{h}\right\|^{2} \leq\left\langle A_{h} u_{h}, u_{h}\right\rangle \leq A^{*}\left\|u_{h}\right\|^{2}, \quad \forall u_{h} \in H_{1, h}, \\
B_{*}\left\|v_{h}\right\|^{2} \leq\left\langle B_{h} v_{h}, v_{h}\right\rangle \leq B^{*}\left\|v_{h}\right\|^{2}, \quad \forall v_{h} \in H_{2, h} ;
\end{gathered}
$$

- bounded operators $P_{h}(r), Q_{h}: H_{1, h} \rightarrow H_{2, h}$ such that there is a constant $Q^{*}>0$ where

$$
\left\|Q_{h}\right\| \leq Q^{*} \text { for all } h>0, \quad P_{h}(r)=r Q_{h}
$$

- discrete source terms $f_{h}^{n+1 / 2} \in H_{1, h}$ and $g_{h}^{n} \in H_{2, h}$; if $(f, g)$ are smooth source terms, then

$$
f_{h}^{n+1 / 2}=S_{1, h} f(\cdot,(n+1 / 2) \Delta t), \quad g_{h}^{n}=S_{2, h} g(\cdot, n \Delta t),
$$

where we define bounded operator $S_{1, h}: \mathcal{H}_{1} \rightarrow H_{1, h}$, and $S_{2, h}: \mathcal{H}_{2} \rightarrow H_{2, h}$ for "sampling" continuum fields onto the spatial grids.

Consider again the acoustic case (related to problem 23). For simplicity we assume we are dealing with rectangular grids, in 2-D for this example, of the form

$$
\mathcal{G}(\mathbf{0}, \mathbf{h})=\left\{\mathbf{x}_{i, j}=(i h, j h): i, j \in \mathbb{Z}\right\}
$$

The simplest staggered-grid finite difference scheme, second-order in time and space, is 
given by

$$
\begin{aligned}
& \frac{1}{\kappa(i h, j h)} \frac{1}{\Delta t}\left[\left(p_{h}\right)_{i, j}^{n+1}-\left(p_{h}\right)_{i, j}^{n}\right]+ \\
& \frac{1}{h}\left[\left(v_{1, h}\right)_{i+1 / 2, j}^{n+1 / 2}-\left(v_{1, h}\right)_{i-1 / 2, j}^{n+1 / 2}+\left(v_{2, h}\right)_{i, j+1 / 2}^{n+1 / 2}-\left(v_{2, h}\right)_{i, j-1 / 2}^{n+1 / 2}\right]=\left(f_{h}\right)_{i, j}^{n+1 / 2} \\
& \rho((i+1 / 2) h, j h) \frac{1}{\Delta t}\left[\left(v_{1, h}\right)_{i+1 / 2, j}^{n+1 / 2}-\left(v_{1, h}\right)_{i+1 / 2, j}^{n-1 / 2}\right]+\frac{1}{h}\left[\left(p_{h}\right)_{i+1, j}^{n}-\left(p_{h}\right)_{i, j}^{n}\right]=\left(g_{1, h}\right)_{i+1 / 2, j}^{n} \\
& \rho(i h,(j+1 / 2) h) \frac{1}{\Delta t}\left[\left(v_{2, h}\right)_{i, j+1 / 2}^{n+1 / 2}-\left(v_{2, h}\right)_{i, j+1 / 2}^{n-1 / 2}\right]+\frac{1}{h}\left[\left(p_{h}\right)_{i, j+1}^{n}-\left(p_{h}\right)_{i, j}^{n}\right]=\left(g_{2, h}\right)_{i, j+1 / 2}^{n}
\end{aligned}
$$

where finite difference solution $\left(p_{h}, v_{1, h}, v_{2, h}\right)$ approximates the continuum fields $\left(p, v_{1}, v_{2}\right)$,

$$
\begin{aligned}
\left(p_{h}\right)_{i, j}^{n} & \approx p(i h, j h, n \Delta t), \\
\left(v_{1, h}\right)_{i+1 / 2, j}^{n+1 / 2} & \approx v_{1}((i+1 / 2) h, j h,(n+1 / 2) \Delta t), \\
\left(v_{2, h}\right)_{i, j+1 / 2}^{n+1 / 2} & \approx v_{2}(i h,(j+1 / 2) h,(n+1 / 2) \Delta t) .
\end{aligned}
$$

We emphasize that the velocity fields for finite difference scheme 25 are staggered with respect to spatial grids, more specifically each component is shifted by half a cell size in its respective axis.

Relating system 25 with 24 , we see

$$
\left(u_{h}\right)_{i, j}=\left(p_{h}\right)_{i, j}, \quad\left(v_{h}\right)_{i, j}=\left[\begin{array}{c}
\left(v_{1, h}\right)_{i+1 / 2, j} \\
\left(v_{2, h}\right)_{i, j+1 / 2}
\end{array}\right]
$$

The space $H_{1, h}$ corresponds to the set of square summable scalar-valued functions on rectangular grids, and $H_{2, h}$ is the set of square summable $\mathbb{R}^{2}$-valued functions on half-cell shifted 
grids, both equipped with a discrete $L^{2}$ inner-products,

$$
\begin{aligned}
& \left\langle u_{h}, \tilde{u}_{h}\right\rangle=h^{2} \sum_{i, j}\left(u_{h}\right)_{i, j}\left(\tilde{u}_{h}\right)_{i, j} \quad \text { for } u_{h}, \tilde{u}_{h} \in H_{1, h} \\
& \left\langle v_{h}, \tilde{v}_{h}\right\rangle=h^{2} \sum_{i, j}\left(v_{h}\right)_{i, j} \cdot\left(\tilde{v}_{h}\right)_{i, j} \quad \text { for } v_{h}, \tilde{v}_{h} \in H_{2, h} .
\end{aligned}
$$

Inner-products and norms of the spaces $\mathcal{H}_{1}, \mathcal{H}_{2}, H_{1, h}, H_{2, h}$ are all denoted by $\langle\cdot, \cdot\rangle$ and $\|\cdot\|$ and interpreted given the context, unless otherwise specified.

Sampling operators $S_{1, h}$ and $S_{2, h}$ coincide with evaluating continuum functions on grid or shifted grid points accordingly,

$$
\left(S_{1, h} u\right)_{i, j}=u(i h, j h), \quad\left(S_{2, h} v\right)_{i, j}=\left[\begin{array}{c}
v_{1}((i+1 / 2) h, j h) \\
v_{2}(i h,(j+1 / 2) h)
\end{array}\right] .
$$

Discretized coefficient operators $A_{h}$ and $B_{h}$ are given by,

$$
\left(A_{h} u_{h}\right)_{i, j}=\frac{1}{\kappa(i h, j h)}\left(u_{h}\right)_{i j}, \quad\left(B_{h} v_{h}\right)_{i, j}=\left[\begin{array}{c}
\rho((i+1 / 2) h, j h)\left(v_{1, h}\right)_{i+1 / 2, j} \\
\rho(i h,(j+1 / 2) h)\left(v_{2, h}\right)_{i, j+1 / 2}
\end{array}\right] .
$$

Lastly, $P_{h}$ and $P_{h}^{T}$ correspond to second-order approximations of the gradient and the negative of the divergence respectively;

$$
\begin{aligned}
\left(P_{h}(r) u_{h}\right)_{i, j} & =r\left[\begin{array}{c}
\left(u_{h}\right)_{i+1, j}-\left(u_{h}\right)_{i, j} \\
\left(u_{h}\right)_{i, j+1}-\left(u_{h}\right)_{i, j}
\end{array}\right] \\
\left(P_{h}(r)^{T} v_{h}\right)_{i, j} & =-r\left[\left(v_{1, h}\right)_{i+1 / 2, j}-\left(v_{1, h}\right)_{i-1 / 2, j}+\left(v_{2, h}\right)_{i, j+1 / 2}-\left(v_{2, h}\right)_{i, j-1 / 2}\right]
\end{aligned}
$$

again, with $r=\Delta t / h$. In general, the differential operator $P$ can be approximated by a 
family of central difference operators $P_{h}$ of even order $2 p$ for $p \in \mathbb{N}$, resulting in the $2-2 p$ staggered-grid finite difference schemes.

\section{Energy Estimates}

Define the following energy form:

$$
E^{n}:=E\left(u_{h}^{n}, v_{h}^{n-1 / 2}\right):=\frac{1}{2}\left(\left\langle A_{h} u_{h}^{n}, u_{h}^{n}\right\rangle+\left\langle B_{h} v_{h}^{n-1 / 2}, v_{h}^{n-1 / 2}\right\rangle+\left\langle P_{h}^{T} v_{h}^{n-1 / 2}, u_{h}^{n}\right\rangle\right) .
$$

Theorem 5. For a solution $\left(u_{h}, v_{h}\right)$ of system 24 with $\left(f_{h}, g_{h}\right) \equiv 0$, it follows that $E^{n}$ is independent of time index $n$. Moreover, for sufficiently small $r$, there exist $0<C_{*} \leq C^{*}$ such that

$$
C_{*}\left(\left\|u_{h}\right\|^{2}+\left\|v_{h}\right\|^{2}\right) \leq E\left(u_{h}, v_{h}\right) \leq C^{*}\left(\left\|u_{h}\right\|^{2}+\left\|v_{h}\right\|^{2}\right) .
$$

Proof. First we show the energy conservation property:

$$
\begin{aligned}
E^{n+1}-E^{n}=\left\langle A_{h}\left(u_{h}^{n+1}-u_{h}^{n}\right),\left(u_{h}^{n+1}+u_{h}^{n}\right)\right\rangle & +\left\langle B_{h}\left(v_{h}^{n+1 / 2}-v_{h}^{n-1 / 2}\right),\left(v_{h}^{n+1 / 2}+v_{h}^{n-1 / 2}\right)\right\rangle \\
& \left.\left.+\left\langle P_{h}^{T} v_{h}^{n+1 / 2}, u_{h}^{n+1}\right\rangle\right)-\left\langle P_{h}^{T} v_{h}^{n-1 / 2}, u_{h}^{n}\right\rangle\right) \\
=\left\langle-P_{h}^{T} v_{h}^{n+1 / 2},\left(u_{h}^{n+1}+u_{h}^{n}\right)\right\rangle & +\left\langle P_{h} u_{h}^{n},\left(v_{h}^{n+1 / 2}+v_{h}^{n-1 / 2}\right)\right\rangle \\
& \left.\left.+\left\langle P_{h}^{T} v_{h}^{n+1 / 2}, u_{h}^{n+1}\right\rangle\right)-\left\langle P_{h}^{T} v_{h}^{n-1 / 2}, u_{h}^{n}\right\rangle\right)=0 .
\end{aligned}
$$

The upper bound in 26 is clear from $h$-uniform bounds on $A_{h}, B_{h}, P_{h}$. The lower bound follows from

$$
E\left(u_{h}, v_{h}\right) \geq A_{*}\left\|u_{h}\right\|^{2}+B_{*}\left\|v_{h}\right\|^{2}-r Q^{*}\left\|u_{h}\right\|\left\|v_{h}\right\|
$$

which is a positive definite form in $\left\|u_{h}\right\|,\left\|v_{h}\right\|$ when $r Q^{*}<\sqrt{A^{*} B^{*}}$. In other words, we 
have established positive definiteness of $E(\cdot, \cdot)$, and more importantly, that the energy form is equivalent to a norm.

\section{$L^{2}$ Convergence of Smooth Solutions}

Given theorem 5 , that is, equivalency of between the $L^{2}$ and energy norms, we focus on proving stability estimates with respect to the energy norm in order to imply $L^{2}$ convergence.

Theorem 6. For $r$ sufficiently small that $E$ is positive definite, stability follows, i.e., there exists $K \geq 0, \lambda>1$ so that

$$
E^{n} \leq \lambda^{n} E^{0}+K h \sum_{m=0}^{n-1} \lambda^{n-m+1}\left(\left\|f_{h}^{m-1 / 2}\right\|^{2}+\left\|g_{h}^{m}\right\|^{2}\right)
$$

where $\left(u_{h}, v_{h}\right)$ satisfy the inhomogeneous system 24 with source terms $\left(f_{h}, g_{h}\right)$.

Proof. The same arithmetic as used in the homogeneous case leads to

$$
\begin{aligned}
E^{n+1}-E^{n} & =r h\left\langle f_{h}^{n+1 / 2}, u_{h}^{n+1}+u_{h}^{n}\right\rangle+r h\left\langle g_{h}^{n}, v_{h}^{n+1 / 2}+v_{h}^{n-1 / 2}\right\rangle \\
& \leq \frac{r h}{2 \alpha^{2}}\left(\left\|f_{h}^{n+1 / 2}\right\|^{2}+\left\|g_{h}^{n}\right\|^{2}\right)+\frac{\alpha^{2} r h}{C_{*}}\left(E^{n+1}+E^{n}\right)
\end{aligned}
$$

for any $\alpha \in(0,1)$. Choose $\alpha$ so that $\alpha^{2} r h<C_{*}$ (that is, can make fixed choice of $\alpha$ for $r h$ small enough), and set

$$
\lambda=\left(1+\frac{\alpha^{2} r h}{C_{*}}\right)\left(1-\frac{\alpha^{2} r h}{C_{*}}\right)^{-1} \quad \text { and } \quad K=\frac{\frac{r}{2 \alpha^{2}}}{\left(1+\frac{\alpha^{2} r h}{C_{*}}\right)}
$$


Then

$$
\begin{gathered}
\lambda^{-1} E^{n+1}-E^{n}=K h\left(\left\|f_{h}^{n+1 / 2}\right\|^{2}+\left\|g_{h}^{n}\right\|^{2}\right) \\
\Longrightarrow \lambda^{-(n+1)} E^{n+1}-\lambda^{-n} E^{n}=\lambda^{-n} K h\left(\left\|f_{h}^{n+1 / 2}\right\|^{2}+\left\|g_{h}^{n}\right\|^{2}\right)
\end{gathered}
$$

whence

$$
\lambda^{-n} E^{n}=E^{0}+K h \sum_{m=0}^{n-1} \lambda^{-m+1}\left(\left\|f_{h}^{m-1 / 2}\right\|^{2}+\left\|g_{h}^{m}\right\|^{2}\right) .
$$

Theorem 7. Suppose that $\left(u^{n}, v^{n-1 / 2}\right) \in \mathcal{H}_{1} \times \mathcal{H}_{2}$, and $\left(u_{h}^{n}, v_{h}^{n-1 / 2}\right)$ solves the discretized system 24 with initial data

$$
u_{h}^{0}=S_{1, h} u^{0}, \quad v_{h}^{-1 / 2}=S_{2, h} v^{-1 / 2} .
$$

Set

$$
\begin{aligned}
\delta f_{h}^{n+1 / 2} & =\frac{1}{r h}\left(A_{h}\left(S_{1, h} u^{n+1}-S_{1, h} u^{n}\right)+P_{h}^{T} S_{2, h} v^{n+1 / 2}\right)+f_{h}^{n+1 / 2} \\
\delta g_{h}^{n} & =\frac{1}{r h}\left(B_{h}\left(S_{2, h} v^{n+1 / 2}-S_{2, h} v^{n-1 / 2}\right)-P_{h} S_{1, h} u^{n}\right)+g_{h}^{n} .
\end{aligned}
$$

Moreover, let $T>0$ be given, independent of $h$. If $\left(\delta f_{h}, \delta g_{h}\right)$, as defined above, satisfy the estimate

$$
\left\|\delta f_{h}^{m-1 / 2}\right\|^{2}+\left\|\delta g_{h}^{m}\right\|^{2} \leq L^{2} h^{2 p}
$$

for $0 \leq m \leq N, N \Delta t=T$, then

$$
E\left(u_{h}^{n}-S_{1, h} u^{n}, v_{h}^{n-1 / 2}-S_{2, h} v^{n-1 / 2}\right) \leq M^{2} L^{2} h^{2 p} .
$$


Proof. Define $K, \lambda$ as in 27, then it follows from previous theorem that

$$
E\left(u_{h}^{n}-S_{1, h} u^{n}, v_{h}^{n-1 / 2}-S_{2, h} v^{n-1 / 2}\right) \leq K h \sum_{m=0}^{n-1} \lambda^{n-m+1}\left(\left\|\delta f_{h}^{m-1 / 2}\right\|^{2}+\left\|\delta g_{h}^{m}\right\|^{2}\right)
$$

which implies

$$
E\left(u_{h}^{n}-S_{1, h} u^{n}, v_{h}^{n-1 / 2}-S_{2, h} v^{n-1 / 2}\right) \leq K L^{2} h^{2 p+1} \frac{\lambda^{N}-1}{\lambda-1}
$$

using estimate 29. Note that if $h$ is sufficiently small, then $\lambda<1+2 \frac{\alpha^{2} r h}{C_{*}}$ so $\lambda^{N} \leq$ $\exp \left(2 \frac{\alpha^{2}}{C_{*}} T\right)$, where $T=N r h$. If $h$ is perhaps smaller yet, then $\lambda>1+\frac{\alpha^{2} r h}{2 C_{*}}$. Putting this all together, there is $M^{2}$ depending on $T$ and all of the other constants in the setup, so that if $n r h<T$ so that 30 holds.

If $(u, v)$ is a smooth solution of the acoustic (pressure-velocity) system 23 with smooth right-hand sides $(f, g)$, and $\left(u_{h}, v_{h}\right)$ is the staggered-grid finite difference approximation of order 2-2p, then, from standard truncation error calculations, for each time slice of compact support we have,

$$
\begin{aligned}
\delta f_{h}^{n+1 / 2} & =O\left(\Delta t^{2}+h^{2 p}\right) \\
\delta g_{h}^{n} & =O\left(\Delta t^{2}+h^{2 p}\right)
\end{aligned}
$$

point-wise and uniformly in $i, j$. Since the support is uniformly bounded if $0 \leq n \Delta t \leq T$, the $O$ statements apply to the $L^{2}$ norms as well. Thus, by equivalency of the energy form with the $L^{2}$-norm, and the corollary above, we can conclude

$$
\left\|u_{h}-S_{1, h} u\right\|+\left\|v_{h}-S_{2, h} v\right\|=O\left(\Delta t^{2}+h^{2 p}\right),
$$


that is, $2-2 p$ order convergence in the $L^{2}$ sense.

\section{Weak Convergence of Singular Solutions}

Let $n_{\min }, n_{\max }$ be integers. Suppose that the grid-function sequence $\left(u_{h}^{n}, v_{h}^{n+1 / 2}\right)$, satisfies 24 with source term $\left(f_{h}^{n+1 / 2}, g_{h}^{n}\right)$ and vanish identically for $n<n_{\min }$. Let $\left(\tilde{u}_{h}^{n+1 / 2}, \tilde{v}_{h}^{n}\right)$ be another sequence of grid functions that vanish for $n>n_{\max }$. Then,

$$
\begin{gathered}
\Delta t \sum_{n}\left(\left\langle\tilde{u}_{h}^{n+1 / 2}, f^{n+1 / 2}\right\rangle+\left\langle\tilde{v}_{h}^{n}, g_{h}^{n}\right\rangle\right) \\
=\sum_{n}\left(\left\langle\tilde{u}_{h}^{n+1 / 2}, A_{h}\left(u_{h}^{n+1}-u_{h}^{n}\right)+P_{h}^{T} v_{h}^{n+1 / 2}\right\rangle+\left\langle\tilde{v}_{h}^{n}, B_{h}\left(v_{h}^{n+1 / 2}-v_{h}^{n-1 / 2}\right)-P_{h} u_{h}^{n}\right\rangle\right) \\
=\sum_{n}\left(\left\langle A_{h}\left(\tilde{u}_{h}^{n-1 / 2}-\tilde{u}_{h}^{n+1 / 2}\right), u_{h}^{n}\right\rangle-\left\langle P_{h} \tilde{u}_{h}^{n+1 / 2}, v_{h}^{n+1 / 2}\right\rangle+\left\langle B_{h}\left(\tilde{v}_{h}^{n}-\tilde{v}_{h}^{n+1}\right), v_{h}^{n+1 / 2}\right\rangle+\left\langle P_{h}^{T} \tilde{v}_{h}^{n}, u_{h}^{n}\right\rangle\right) \\
=\sum_{n}\left(\left\langle A_{h}\left(\tilde{u}_{h}^{n-1 / 2}-\tilde{u}_{h}^{n+1 / 2}\right)-P_{h}^{T} \tilde{v}_{h}^{n}, u_{h}^{n}\right\rangle+\left\langle B_{h}\left(\tilde{v}_{h}^{n}-\tilde{v}_{h}^{n+1}\right)+P_{h} \tilde{u}_{h}^{n+1 / 2}, v_{h}^{n+1 / 2}\right\rangle\right.
\end{gathered}
$$

Define $\left(\tilde{f}_{h}^{n}, \tilde{g}_{h}^{n+1 / 2}\right) \in H_{1, h} \times H_{2, h}$ by

$$
\begin{aligned}
A_{h}\left(\tilde{u}_{h}^{n-1 / 2}-\tilde{u}_{h}^{n+1 / 2}\right)-P_{h}^{T} \tilde{v}^{n} & =r h \tilde{f}_{h}^{n} \\
B_{h}\left(\tilde{v}^{n}-\tilde{v}^{n+1}\right)+P_{h} \tilde{u}^{n+1 / 2} & =\operatorname{rh} \tilde{g}_{h}^{n+1 / 2} .
\end{aligned}
$$

Then, identity 31 can be re-written as

$$
\Delta t \sum_{n}\left(\left\langle\tilde{u}_{h}^{n+1 / 2}, f_{h}^{n+1 / 2}\right\rangle+\left\langle\tilde{v}_{h}^{n}, g_{h}^{n}\right\rangle\right)=\Delta t \sum_{n}\left(\left\langle\tilde{f}_{h}^{n}, u_{h}^{n}\right\rangle+\left\langle\tilde{g}_{h}^{n+1 / 2}, v_{h}^{n+1 / 2}\right\rangle\right) .
$$

Now specialize to the acoustic case - the elastic case is similar. In this case $g$ (and 
$\tilde{g}$ ) is vector-valued, and $f$ (and $\tilde{f}$ ) is scalar-valued. Once again, $P_{h}$ is a discrete gradient; assume that it is accurate of order $2 p$, as before. $P_{h}^{T}$ is a discrete (negative) divergence, also accurate of order $2 p$. The adjoint system 32 is recognized as another discretization of the acoustic system 23, with two differences: the time index is dual to the one used in 24 , that is, shifted by $\Delta t / 2$, and the inhomogenous terms get a negative sign. Evidently, the truncation error analysis is exactly the same.

Suppose that $(\tilde{f}, \tilde{g})$ are smooth in both temporal and spatial variables, and that

$$
\tilde{f}_{h}^{n}=S_{1, h} \tilde{f}^{n}, \quad \tilde{g}_{h}^{n+1 / 2}=S_{2, h} \tilde{g}^{n+1 / 2} .
$$

Denote by $(\tilde{u}, \tilde{v})$ the (weak) solution of the acoustic system 23 with right-hand sides $(-\tilde{f},-\tilde{g})$ that vanishes for $t>T$. The standard theory for hyperbolic systems shows that $(\tilde{u}, \tilde{v})$ is also smooth. Let $\left(\tilde{u}_{h}^{n+1 / 2}, \tilde{v}_{h}^{n}\right)$ be the solution of the discrete adjoint system 32 with right-hand sides $\left(\tilde{f}_{h}^{n}, \tilde{g}_{h}^{n+1 / 2}\right)$. Then (as before assuming that $P_{h}$ is accurate of order $2 p$ )

$$
\delta \tilde{u}_{h}^{n+1 / 2}=\tilde{u}_{h}^{n+1 / 2}-S_{1, h} \tilde{u}^{n+1 / 2}, \quad \delta \tilde{v}_{h}^{n}=\tilde{v}_{h}^{n}-S_{2, h} \tilde{v}^{n}
$$

solve 32 with right-hand side that is $O\left(\Delta t^{2}+h^{2 p}\right)$, so according to the error estimate 30 (which applies ipso facto to the system 32),

$$
\left\|\delta \tilde{u}_{h}^{n+1 / 2}\right\|^{2}+\left\|\delta \tilde{v}_{h}^{n}\right\|^{2}=O\left(\Delta t^{2}+h^{2 p}\right)
$$

over any finite range of $n \Delta t$.

We now have all of the ingredients to prove weak convergence, which we now state: 
Theorem 8. Let $(u, v)$ be a solution to continuum problem 21 with singular source terms $(f, g)$ of the form

$$
\begin{aligned}
& f(\mathbf{x}, t)=w(t) D^{\mathbf{s}_{1}} \delta\left(\mathbf{x}-\mathbf{x}^{*}\right), \\
& g(\mathbf{x}, t)=z(t) D^{\mathbf{s}_{2}} \delta\left(\mathbf{x}-\mathbf{x}^{*}\right),
\end{aligned}
$$

where $w(t) \in \mathbb{R}^{k_{1}}$ and $z(t) \in \mathbb{R}^{k_{2}}$ are smooth vector-valued functions in time. Also, let $\left(u_{h}, v_{h}\right)$ denote the corresponding finite difference solution with singular source approximates $\left(f_{h}, g_{h}\right)$ of order $q$. Then, for any smooth test functions $(\tilde{f}, \tilde{g})$, we have the following error estimate

$$
\begin{aligned}
\mathcal{E} & :=\left|\int_{0}^{T}\{\langle u, \tilde{f}\rangle+\langle v, \tilde{g}\rangle\} d t-\Delta t \sum_{n=0}^{N}\left\{\left\langle u_{h}^{n}, \tilde{f}_{h}^{n}\right\rangle+\left\langle v_{h}^{n+1 / 2}, \tilde{g}_{h}^{n+1 / 2}\right\rangle\right\}\right| \\
& =O\left(\Delta t^{2}+h^{q}+\left(\Delta t^{2}+h^{2 p}\right) h^{-N^{*}-d / 2}\right),
\end{aligned}
$$

with $N^{*}=\max \left\{\left|\mathbf{s}_{1}\right|,\left|\mathbf{s}_{2}\right|\right\}$. Again, $\left(\tilde{f}_{h}, \tilde{g}_{h}\right)$ as given by 34.

Proof. Using 33, and its continuum version, error $\mathcal{E}$ is rewritten in terms of inner products with the singular source terms, both in the continuum and discrete sense, i.e.,

$$
=\left|\int_{0}^{T}\{\langle f, \tilde{u}\rangle+\langle g, \tilde{v}\rangle\} d t-\Delta t \sum_{n=0}^{N}\left\{\left\langle f_{h}^{n+1 / 2}, \tilde{u}_{h}^{n+1 / 2}\right\rangle+\left\langle g_{h}^{n}, \tilde{v}_{h}^{n}\right\rangle\right\}\right| .
$$

Note $(\tilde{p}, \tilde{v})$ are solutions to problem 21 with smooth source terms $(-\tilde{f},-\tilde{g})$, and $\left(\tilde{u}_{h}, \tilde{v}_{h}\right)$ is the respective staggered grid finite difference solutions with sources $\left(-\tilde{f}_{h},-\tilde{g}_{h}\right)$. Further- 
more,

$$
\begin{aligned}
\mathcal{E} & =\left|\int_{0}^{T}\{\langle f, \tilde{u}\rangle+\langle g, \tilde{v}\rangle\} d t-\Delta t \sum_{n=0}^{N}\left\{\left\langle f_{h}^{n+1 / 2}, \tilde{u}_{h}^{n+1 / 2} \pm S_{1, h} \tilde{u}^{n+1 / 2}\right\rangle+\left\langle g_{h}^{n}, \tilde{v}_{h}^{n} \pm S_{2, h} \tilde{v}^{n}\right\rangle\right\}\right| \\
& \leq \mathcal{E}_{1}+\mathcal{E}_{2}
\end{aligned}
$$

with

$$
\begin{gathered}
\mathcal{E}_{1}:=\left|\int_{0}^{T}\{\langle f, \tilde{u}\rangle+\langle g, \tilde{v}\rangle\} d t-\Delta t \sum_{n=0}^{N}\left\{\left\langle f_{h}^{n+1 / 2}, S_{1, h} \tilde{u}^{n+1 / 2}\right\rangle+\left\langle g_{h}^{n}, S_{2, h} \tilde{v}^{n}\right\rangle\right\}\right| \\
\mathcal{E}_{2}:=\left|\Delta t \sum_{n=0}^{N}\left\{\left\langle f_{h}^{n+1 / 2}, \delta \tilde{u}_{h}^{n+1 / 2}\right\rangle+\left\langle g_{h}^{n}, \delta \tilde{v}_{h}^{n}\right\rangle\right\}\right| .
\end{gathered}
$$

For $\mathcal{E}_{1}$, we first focus on the terms involving $f$ and $\tilde{u}$;

$$
\begin{array}{r}
\left|\int_{0}^{T}\langle f, \tilde{u}\rangle d t-\Delta t \sum_{n=0}^{N}\left\langle f_{h}^{n+1 / 2}, S_{1, h} \tilde{u}^{n+1 / 2}\right\rangle\right| \leq \\
\left|\int_{0}^{T}\langle f, \tilde{u}\rangle d t-\Delta t \sum_{n=0}^{N}\left\langle f^{n+1 / 2}, \tilde{u}^{n+1 / 2}\right\rangle\right|+\left|\Delta t \sum_{n=0}^{N}\left\{\left\langle f_{h}^{n+1 / 2}, S_{1, h} \tilde{u}^{n+1 / 2}\right\rangle-\left\langle f^{n+1 / 2}, \tilde{u}^{n+1 / 2}\right\rangle\right\}\right|,
\end{array}
$$

which is nothing more than quadrature error for the time integration and the singular source discretization error. In particular,

$$
\left|\int_{0}^{T}\langle f, \tilde{u}\rangle d t-\Delta t \sum_{n=0}^{N}\left\langle f^{n+1 / 2}, \tilde{u}^{n+1 / 2}\right\rangle\right|=O\left(\Delta t^{2}\right)
$$

from standard error estimates of the midpoint rule, and

$$
\left|\Delta t \sum_{n=0}^{N}\left\{\left\langle f_{h}^{n+1 / 2}, S_{1, h} \tilde{u}^{n+1 / 2}\right\rangle-\left\langle f^{n+1 / 2}, \tilde{u}^{n+1 / 2}\right\rangle\right\}\right|=O\left(h^{q}\right)
$$

from the singular source approximation theory (theorem 2). Similar error estimates can be 
derived for the terms involving $g$ and $\tilde{v}{ }^{1}$ We bound $\mathcal{E}_{2}$ using $L^{2}$-error estimates of finite difference solutions for smooth problems, and using $L^{2}$-bounds of the discrete singular source approximations; one can show

$$
\left\|f_{h}\right\|_{2}=O\left(h^{-\left|\mathbf{s}_{1}\right|-d / 2}\right) \quad \text { and } \quad\left\|g_{h}\right\|_{2}=O\left(h^{-\left|\mathbf{s}_{2}\right|-d / 2}\right) .
$$

Whence,

$$
\mathcal{E}_{2} \leq \Delta t \sum_{n=0}^{N}\left\{\left\|f_{h}^{n+1 / 2}\right\|_{2}\left\|\delta \tilde{u}_{h}^{n+1 / 2}\right\|_{2}+\left\|g_{h}^{n}\right\|_{2}\left\|\delta \tilde{v}_{h}^{n}\right\|_{2}\right\}=O\left(\left(\Delta t^{2}+h^{2 p}\right) h^{-N^{*}-d / 2}\right)
$$

Estimate 36 thus follows.

Consider the simpler case where only multipoles of order zero are present, that is $N^{*}=0$. According to estimate 36 , we have weak convergence at rate $2-d / 2$ if we choose $q=2 p$ and $\Delta t=O(h)$. In other words, convergence (in the weak sense) is guaranteed though at a suboptimal rate. For multipoles of order $N^{*}>0$, to retrieve the smooth solution behavior, we would require $2 p>N^{*}+d / 2$ and $\Delta t \rightarrow 0$ like a positive power of $h$.

The (weak) error estimate we present here can be improved by making the following observation: the $h^{-d / 2}$ factor originates from $L^{2}$-bounds of multipole source approximations. In particular, we can remove this factor by using $L^{1}$-bounds instead, mainly

$$
\left\|f_{h}\right\|_{1}=O\left(h^{-\left|\mathbf{s}_{1}\right|}\right) \quad \text { and } \quad\left\|g_{h}\right\|_{1}=O\left(h^{-\left|\mathbf{s}_{2}\right|}\right) .
$$

\footnotetext{
${ }^{1} O\left(\Delta t^{2}\right)$ quadrature error estimates follow from noting that the summation term coincides with the trapezoidal rule, assuming that $g(t)$ and $\tilde{v}(t)$ satisfy homogenous initial and final time conditions respectively.
} 
Applying Hölder's inequality to $\mathcal{E}_{2}$ yields

$$
\mathcal{E}_{2} \leq \Delta t \sum_{n=0}^{N}\left\{\left\|f_{h}^{n+1 / 2}\right\|_{1}\left\|\delta \tilde{u}_{h}^{n+1 / 2}\right\|_{\infty}+\left\|g_{h}^{n}\right\|_{1}\left\|\delta \tilde{v}_{h}^{n}\right\|_{\infty}\right\}=O\left(\left(\Delta t^{2}+h^{2 p}\right) h^{-N^{*}}\right)
$$

We conjecture point-wise bounds hold for smooth $\delta \tilde{u}$ and $\delta \tilde{v}$, whence

$$
\mathcal{E}=O\left(\Delta t^{2}+h^{q}+\left(\Delta t^{2}+h^{2 p}\right) h^{-N^{*}}\right),
$$

yielding optimal rates for the $N^{*}=0$ case, again if $q \geq 2 p$. Note that 37 follows through if $L^{\infty}$ error terms $\left\|\delta \tilde{u}_{h}\right\|_{\infty}$ and $\left\|\delta \tilde{v}_{h}\right\|_{\infty}$ have optimal rates. The authors are not, however, aware of any such $L^{\infty}$ error estimates for the types of hyperbolic systems considered here. One could potentially attempt to prove stability in $L^{\infty}$ in order to apply the Lax equivalence theorem and imply $L^{\infty}$ estimates (see Brenner et al. (2006)) or alternatively proof $L^{\infty}$ estimates requiring only $L^{2}$ stability (see Layton (1982)). 


\section{NUMERICAL TESTS AND RESULTS}

We have implemented the singular source approximation as discussed in the theory section using the $\mathrm{C}++$ packages IWave and Rice Vector Library (RVL); (Symes et al., 2011; Padula et al., 2009). The IWave package is a framework for finite difference solvers over uniform grids while the RVL package provides a system of classes for expression of gradient-based optimization algorithms over Hilbert spaces. IWave and RVL come together to form a modeling engine for seismic inversion and migration. Implementation of multipole sources as RVL objects enables straightforward composition with IWave solvers and inclusion in inversion algorithms powered by RVL optimization code. Any other wave equation solver wrapped in the appropriate RVL interfaces could be coupled to the multipole source objects in the same way.

A convergence rate study is performed to corroborate theoretical results pertaining to the accuracy of moment-consistent approximations to multipole sources. In particular, our numerical experiments explore the semi-discrete error of staggered-grid finite difference solutions (time discretization errors are minimized by taking sufficiently small time steps). We used the IWave implementation of staggered-grid finite difference schemes for the acoustic system 23 (Virieux, 1984), of order 2 in time and orders 2 and 4 in space - we refer to these as the 2-2 and 2-4 order schemes respectively. In these experiments we use only scalar (pressure) sources and pressure trace data. Similar results are obtained with other choices. Boundary conditions are of PML type, as described by Hu et al. (2007),

The numerical experiments carried out concern multipoles in 2-D of the form

$$
f(\mathbf{x}, t)=w(t) D^{\mathbf{s}} \delta\left(\mathbf{x}-\mathbf{x}^{*}\right)
$$


for $\mathbf{s}=(0,0),(0,1),(0,2)$. The discretizations of $D^{\mathbf{s}} \delta$ are chosen as to achieve a target order of convergence for the difference schemes, in most cases the nominal spatial order ( $q=2$ for the $2-2$ scheme, $q=4$ for the $2-4$ scheme). Again, the time step is fixed small enough that the time discretization error plays essentially no role in the global error - it reflects truncation error of the spatial derivative and the source approximation only. Timedependent function $w(t)$ is chosen to be a Ricker wavelet with peak frequency of $5 \mathrm{~Hz}$, see figure 3a.

We approximate the convergence rate $R(\mathbf{x})$ at a given location $\mathbf{x}$ via Richardson extrapolation,

$$
R(\mathbf{x})=\log _{2}\left(\frac{\left\|p_{h}(\mathbf{x}, \cdot)-p_{h / 2}(\mathbf{x}, \cdot)\right\|}{\left\|p_{h / 2}(\mathbf{x}, \cdot)-p_{h / 4}(\mathbf{x}, \cdot)\right\|}\right)
$$

where $p_{h}$ denotes the computed pressure field via finite difference using a grid size $h$ respectively. The norm $\|\cdot\|$ is chosen to be

$$
\|p(\mathbf{x}, \cdot)\|:=\sqrt{\Delta t \sum_{k}\left|p\left(\mathbf{x}, t_{k}\right)\right|^{2}}
$$

Coordinates are aligned such that $\mathbf{x}=(z, x)$, where $z$ and $x$ refer to depth and horizontal distance respectively. The computational domain consists of a $4 \mathrm{~km}$ by $4 \mathrm{~km}$ square medium with a constant bulk modulus of $9 G P a$ and buoyancy of $1 \mathrm{~cm}^{3} / \mathrm{kg}$, thus a constant velocity of $3 \mathrm{~km} / \mathrm{s}$. The source is placed slightly off of the center, $\left(z^{*}, x^{*}\right)=(-2003 \mathrm{~m}, 2003 \mathrm{~m})$, as to not coincide with a grid point for any of the computational uniform grids. The following are some other specifications that apply to all tests carried out here:

- total recording time is $1.5 \mathrm{~s}$; 
- spatial grid sizes $h=40 m, h / 2=20 m, h / 4=10 m$;

- time step $\Delta t=0.5 m s$;

Note that the coarsest grid cell size is $40 \mathrm{~m}$ which implies that at least 15 grid points per wavelength (gpw) at peak frequency of $5 \mathrm{~Hz}$ or $5 \mathrm{gpw}$ at $15 \mathrm{~Hz}$ (see figure $3 \mathrm{~b}$ ), in all numerical experiments, thus minimizing the effects of grid dispersion on approximated convergence rates.

\section{Results}

Figures $4 \mathrm{a}-4 \mathrm{c}$ plot a snapshot of the computed pressure field for different multipole sources. Consistent with analytical formulas for the homogeneous unbounded medium case, the observed pressure field exhibited a polarity reversal (or lack of), a symmetry about the $x$-axis (i.e., $z=2003$ ), and an overall decrease in amplitude dependent on the multipole.

Convergence rates are plotted for the $\mathbf{s}=(0,0)$ case in figure 5 , over the entire domain and at a particular depth of $z=2000$ in the left and right column plots respectively. The first row of graphs shows results when using the 2-2 finite difference scheme and the secondorder source approximation. We observe that second order convergence is indeed achieved away from the source. Similarly, fourth order convergence results are observed when using the 2-4 finite difference scheme and the fourth-order source approximation, i.e., second row in figure 5. Lastly, the third row of plots demonstrates the negative effects of using a lower order source approximation relative to the spatial finite difference order, namely a secondorder source approximation with a fourth-order method in space. Clearly, fourth order rates are not achieved and moreover, there seems to be regions where rates dip below or above 
the expected second order. Convergence results for $\mathbf{s}=(0,1)$ and $\mathbf{s}=(0,2)$ are given in figures 6 and 7 respectively with similar results.

\section{DISCUSSION}

Numerical results presented here validate the accuracy of moment-consistent singular source discretizations for controlling the propagation of finite difference truncation error for multipole sources. In particular, optimal spatial convergence rates for the 2-2 and 2-4 staggered grid finite difference methods are achieved, point-wise in space away from source location, when the source approximation order is equal to that of the spatial finite difference approximation order.

The onset distance of optimal order convergence is consistent with the support of the source approximation for the coarsest grid used in the convergence study, that is, $h(q+|\mathbf{s}|)$ with $h=40 \mathrm{~m}$. The erratic behavior of numerical convergence rates within the support of source approximations can be explained in part by how convergence rates were approximated. Namely, numerical solutions over consecutively refined grids are essentially compared, though over the coarsest grid. It follows that the support of $f_{h}$, the source approximation with respect to grid with cell size $h$, will contain the support of $f_{h / 2}$ and a region in the $h$-grid outside the direct influence of $f_{h / 2}$, resulting in irregular convergence rates.

Work by Petersson et al. (2016) demonstrated that optimal convergence rates can be achieved if the source discretization satisfied moment as well as smoothness conditions. Again, their work was centered around central difference approximations of the 1-D ad- 
vection equation. As our numerical tests demonstrate, smoothness conditions were not necessary here, which is attributed to the fact that we use staggered-grid finite difference schemes. In particular, the central finite difference operator used in Petersson et al. (2016) have an associated grid spacing of $2 h$ with respect to an $h$-sized grid, coupled with a source approximation of narrow support, i.e., $2 \epsilon=(q+|\mathbf{s}|) h$, results in the triggering of spurious modes unresolved by the numerical solution. The finite difference operators used here however have a grid spacing of $h$ given that they approximate derivatives over staggered grids. Thus, in essence our narrow source approximations "seem" twice as wide, and therefore smoother, from the point of view of the difference operator $P_{h}$. For this reason, smoothness conditions are not necessary for staggered-grid finite difference schemes.

\section{CONCLUSION}

In this paper we have covered the singular source approximation theory based on moment conditions, which essentially has approximations mimic the behavior of the target distribution $D^{\mathbf{s}} \delta\left(\mathbf{x}-\mathbf{x}^{*}\right)$ on polynomials. Moreover, we give explicit forms of source approximations with narrow support, based on the discrete moment conditions in the context of finite difference solvers: diameter $2 \epsilon=(q+|\mathbf{s}|) h$ for a multipole of order $|\mathbf{s}|$ over a grid of size $h$. As a new result, we connect the discrete and continuum singular source approximation theory by proving that continuum functions generated from the discrete moment conditions indeed satisfy the continuum moment conditions.

Our main contribution was the development of a weak convergence theory that is applicable to a large set of wave propagation problems (including acoustics and elasticity in first-order form) solved via a family of staggered-grid finite difference schemes, larger than 
what is reported in the current literature. Posing the convergence mode of numerical solutions in terms of weak convergence was indeed a natural choice given that source terms are derivatives of the Dirac delta function, that is, distributions. Numerical results, however, give evidence of stronger convergence, namely optimal convergence rates given by numerical scheme under smooth conditions, point-wise away from source location for appropriate source discretizations and in particular for multipoles of order $|\mathbf{s}|=0,1$ and 2 .

\section{ACKNOWLEDGEMENTS}

We are grateful to the sponsors of The Rice Inversion Project for their long-term support, and to the Rice Graduate Education for Minorities (RGEM) and XSEDE scholarship programs for their support of M. Bencomo's Ph.D. research. This material is also based upon work supported by the National Science Foundation under Grant No. DMS-1439786 while the author was in residence at the Institute for Computational and Experimental Research in Mathematics in Providence, RI, during the Fall 2017 semester. 


\section{REFERENCES}

Blazek, K., C. C. Stolk, and W. Symes, 2013, A mathematical framework for inverse wave problems in heterogeneous media: Inverse Problems, 29, 065001:1-34.

Brenner, P., V. Thomée, and L. B. Wahlbin, 2006, Besov spaces and applications to difference methods for initial value problems: Springer, 434.

Cohen, G. C., 2002, Higher order numerical methods for transient wave equations: Springer.

Courant, R., and D. Hilbert, 1962, Methods of mathematical physics: Wiley-Interscience, II.

Epanomeritakis, I., V. Akcelik, O. Ghattas, and J. Bielak, 2009, A Newton-cg method for large-scale three-dimensional elastic full-waveform seismic inversion: Inverse Problems, 24, 24:034015 (26pp).

Hörmander, L., 1969, Linear partial differential operators, 3rd ed.: Springer Verlag.

Hosseini, B., N. Nigam, and J. M. Stockie, 2016, On regularizations of the dirac delta distribution: Journal of Computational Physics, 305, 423-447.

Hu, W., A. Abubakar, and T. Habashy, 2007, Application of the nearly perfectly matched layer in acoustic wave modeling: Geophysics, 72, SM169-SM176.

Komatisch, D., C. Barnes, and J. Tromp, 2000, Simulation of anisotropic wave propagation based upon a spectral element method: Geophysics, 65, 1251-1260.

Königsberger, K., 2013, Analysis 2: Springer-Verlag.

Layton, W. J., 1982, Simplified $l^{\infty}$ estimates for difference schemes for partial differential equations: Proceedings of the American Mathematical Society, 86, 491-495.

Moczo, P., J. O. A. Robertsson, and L. Eisner, 2006, The finite-difference time-domain method for modeling of seismic wave propagation: Advances in Geophysics, 48, 421-516.

Padula, A. D., W. Symes, and S. D. Scott, 2009, A software framework for the abstract 
expression of coordinate-free linear algebra and optimization algorithms: ACM Transactions on Mathematical Software, 36, 8:1-8:36.

Peskin, C. S., 2002, The immersed boundary method: Acta numerica, 11, 479-517.

Petersson, N. A., O. O'Reilly, B. Sjögreen, and S. Bydlon, 2016, Discretizing singular point sources in hyperbolic wave propagation problems: Journal of Computational Physics, 321, $532-555$.

Petersson, N. A., and B. Sjögreen, 2010, Stable grid refinement and singular source discretization for seismic wave simulations: Comm. Comput. Phys., 8, 1074-1110.

Shearer, P. M., 2009, Introduction to seismology: Cambridge University Press.

Symes, W., D. Sun, and M. Enriquez, 2011, From modelling to inversion: designing a well-adapted simulator: Geophysical Prospecting, 59, 814-833. (DOI:10.1111/j.13652478.2011.00977.x).

Tornberg, A., and B. Engquist, 2003, Regularization techniques for numerical approximation of pdes with singularities: J. Sci. Comput., 19, 527-552.

— 2004 , Numerical approximation of singular source terms in differential equations: J. Comp. Phys., 200, 462-488.

Virieux, J., 1984, SH-wave propagation in heterogeneous media: Velocity stress finitedifference method: Geophysics, 49, 1933-1957.

Waldén, J., 1999, On the approximation of singular source terms in differential equations: Numerical Methods for Partial Differential Equations, 15, 503-520.

Yilmaz, O., 2001, Seismic data processing, in Investigations in Geophysics No. 10: Society of Exploration Geophysicists. 


\section{LIST OF FIGURES}

1 Plots of $\eta^{\epsilon}(x), 1-\mathrm{D}$ approximations to $D^{s} \delta(x)$ with $h=1$. (a) $s=0$ and $q=2$, (b) $s=0$ and $q=4,(\mathrm{c}) s=1$ and $q=2$, (d) $s=1$ and $q=4$, (e) $s=2$ and $q=2$, (f) $s=2$ and $q=4$.

2 Plots of $\eta^{\epsilon}(\mathbf{x}), 2-\mathrm{D}$ approximations to $D^{\mathbf{s}} \delta(\mathbf{x})$ with $\mathbf{h}=(1,1)$. (a) $\mathbf{s}=(0,0)$ and $q=2,(\mathrm{~b}) \mathbf{s}=(0,0)$ and $q=4,(\mathrm{c}) \mathbf{s}=(0,1)$ and $q=2,(\mathrm{~d}) \mathbf{s}=(0,1)$ and $q=4$, (e) $\mathbf{s}=(0,2)$ and $q=2$, (f) $\mathbf{s}=(0,2)$ and $q=4$.

3 Ricker wavelet with peak frequency $5 H z$ : (a) time plot, (b) power spectrum plot.

4 Snapshot of pressure field $(t=0.748 \mathrm{sec})$ computed using 2-2 finite difference scheme $(h=10 \mathrm{~m}, \Delta t=0.5 \mathrm{~ms})$ and second-order approximation of multipole source $D^{\mathbf{s}} \delta\left(\mathbf{x}-\mathbf{x}^{*}\right):(\mathrm{a}) \mathbf{s}=(0,0),(\mathrm{b}) \mathbf{s}=(0,1)$, and $(\mathrm{c}) \mathbf{s}=(0,2)$.

5 Convergence rate results for $\mathbf{s}=(0,0)$. Using 2-2 FD scheme and second-order source approximation (a), 2-4 FD scheme and fourth-order source approximation (c), 2-4 FD scheme and second-order source approximation (e). Plots on right column correspond to convergence rates at $z=2000$, related to the left column.

6 Convergence rate results for $\mathbf{s}=(0,1)$. Using 2-2 FD scheme and second-order source approximation (a), 2-4 FD scheme and fourth-order source approximation (c), 2-4 FD scheme and second-order source approximation (e). Plots on right column correspond to convergence rates at $z=2000$, related to the left column.

7 Convergence rate results for $\mathbf{s}=(0,2)$. Using $2-2 \mathrm{FD}$ scheme and second-order source approximation (a), 2-4 FD scheme and fourth-order source approximation (c), 2-4 FD scheme and second-order source approximation (e). Plots on right column correspond to convergence rates at $z=2000$, related to the left column. 


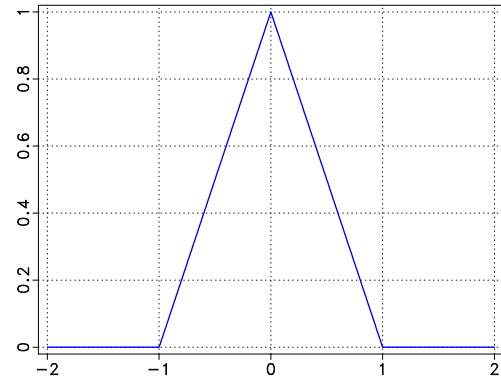

(a)

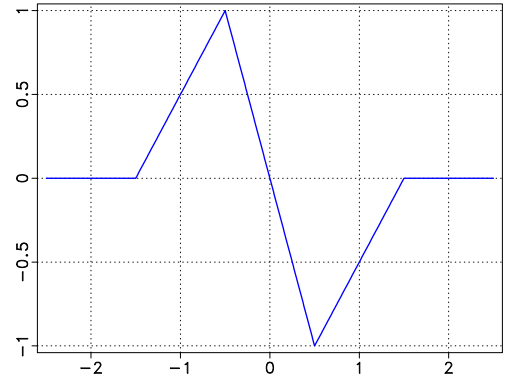

(c)

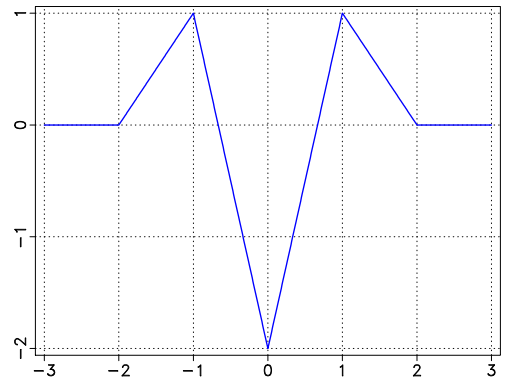

(e)

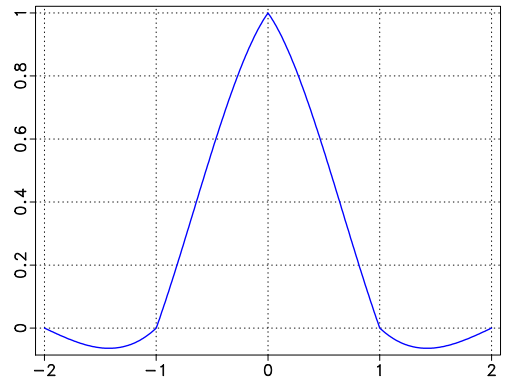

(b)

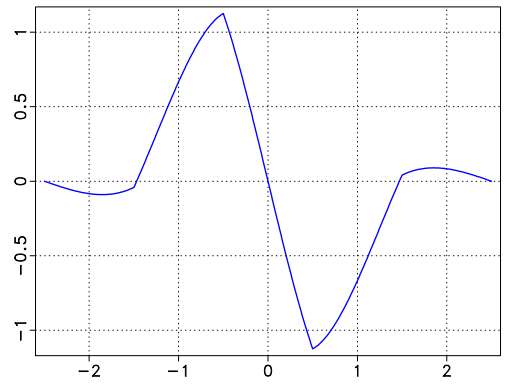

(d)

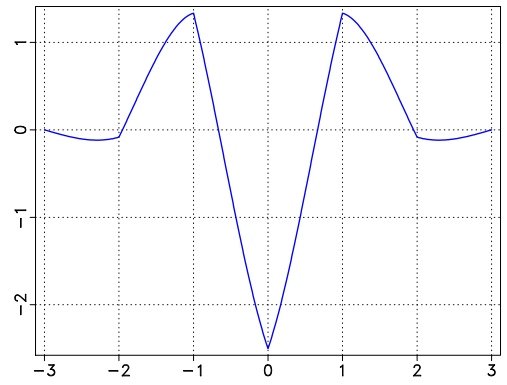

(f)

Figure 1: Plots of $\eta^{\epsilon}(x), 1-\mathrm{D}$ approximations to $D^{s} \delta(x)$ with $h=1$. (a) $s=0$ and $q=2$, (b) $s=0$ and $q=4$, (c) $s=1$ and $q=2$, (d) $s=1$ and $q=4$, (e) $s=2$ and $q=2$, (f) $s=2$ and $q=4$. 


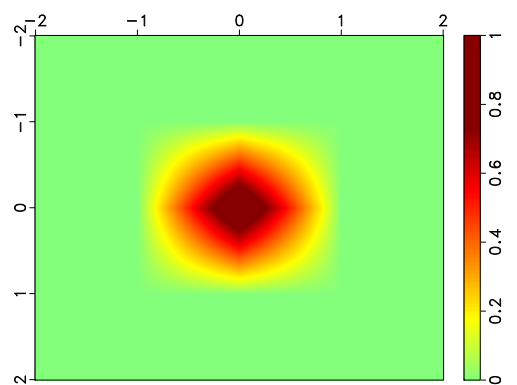

(a)

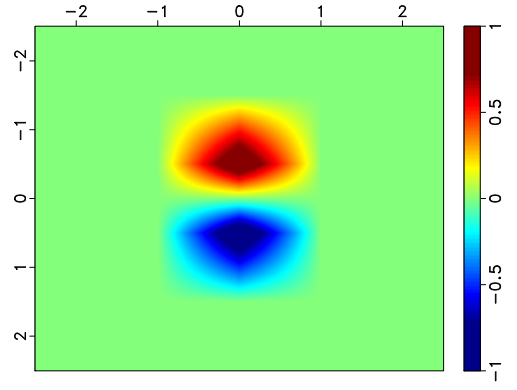

(c)

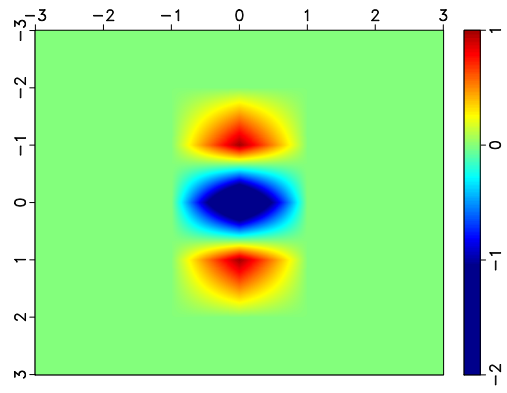

(e)

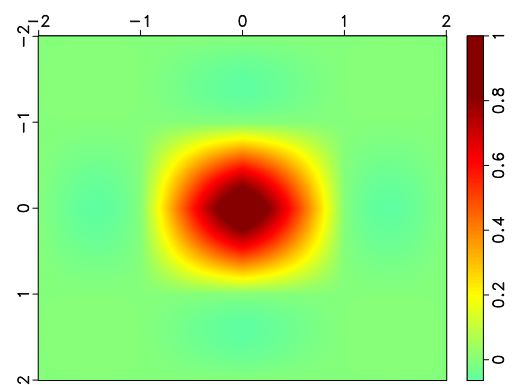

(b)

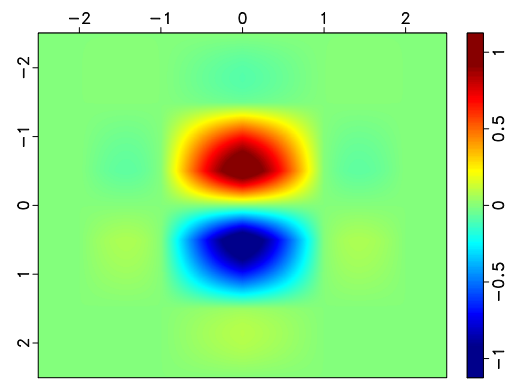

(d)

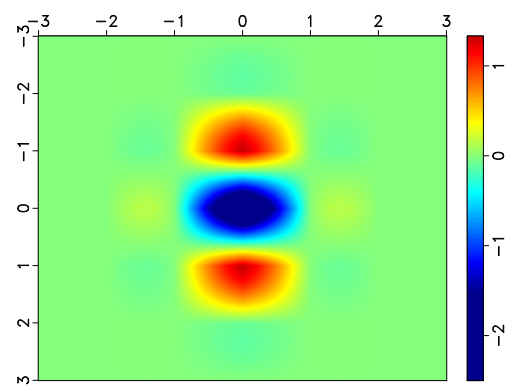

(f)

Figure 2: Plots of $\eta^{\epsilon}(\mathbf{x}), 2-\mathrm{D}$ approximations to $D^{\mathbf{s}} \delta(\mathbf{x})$ with $\mathbf{h}=(1,1)$. (a) $\mathbf{s}=(0,0)$ and $q=2,(\mathrm{~b}) \mathbf{s}=(0,0)$ and $q=4,(\mathrm{c}) \mathbf{s}=(0,1)$ and $q=2,(\mathrm{~d}) \mathbf{s}=(0,1)$ and $q=4$, (e) $\mathbf{s}=(0,2)$ and $q=2$, (f) $\mathbf{s}=(0,2)$ and $q=4$. 


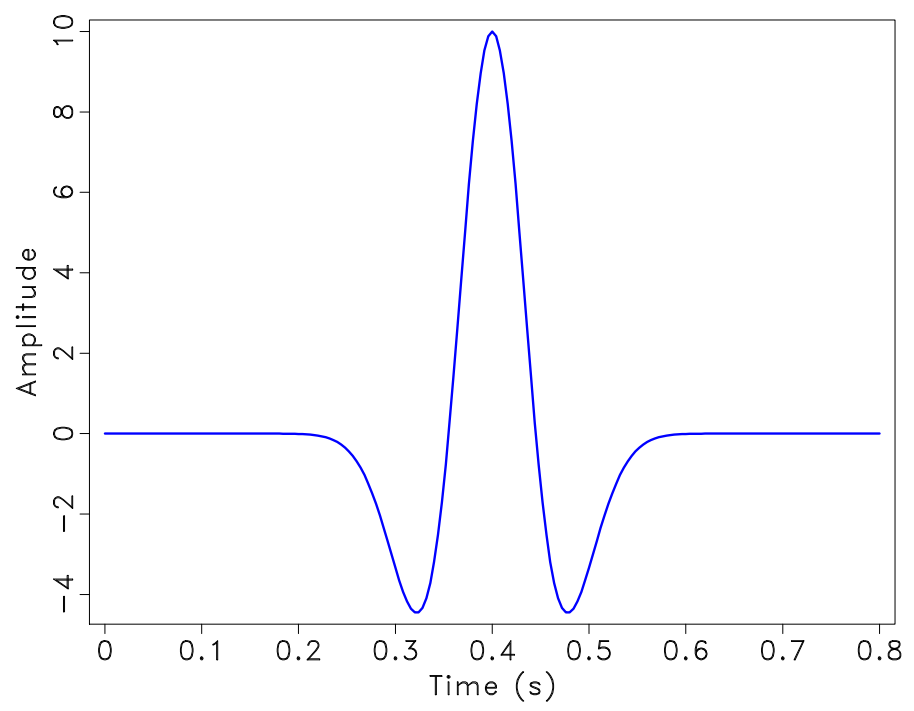

(a)

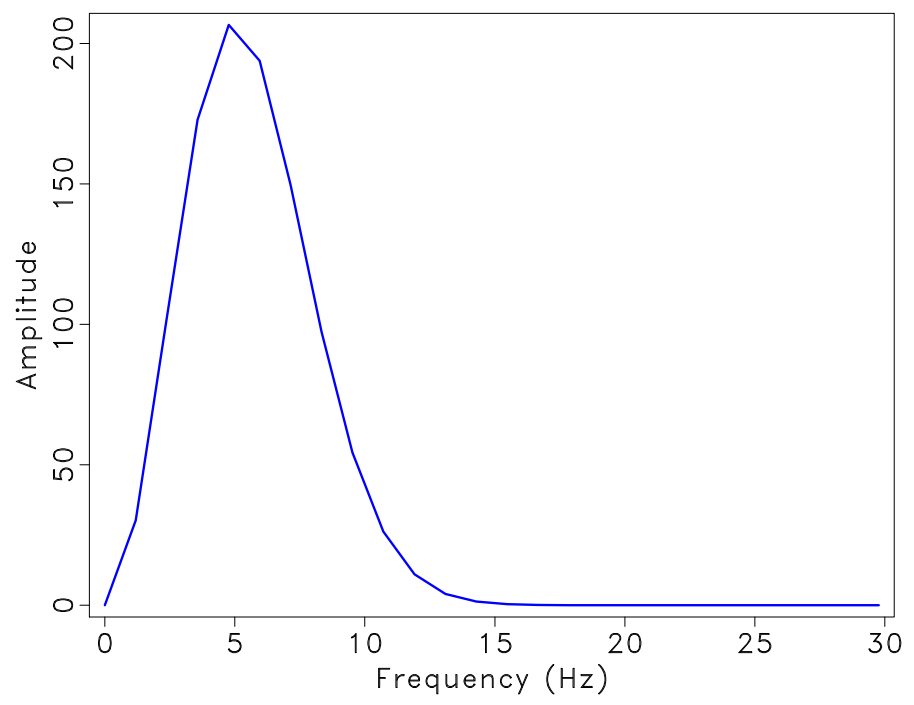

(b)

Figure 3: Ricker wavelet with peak frequency $5 H z$ : (a) time plot, (b) power spectrum plot. 


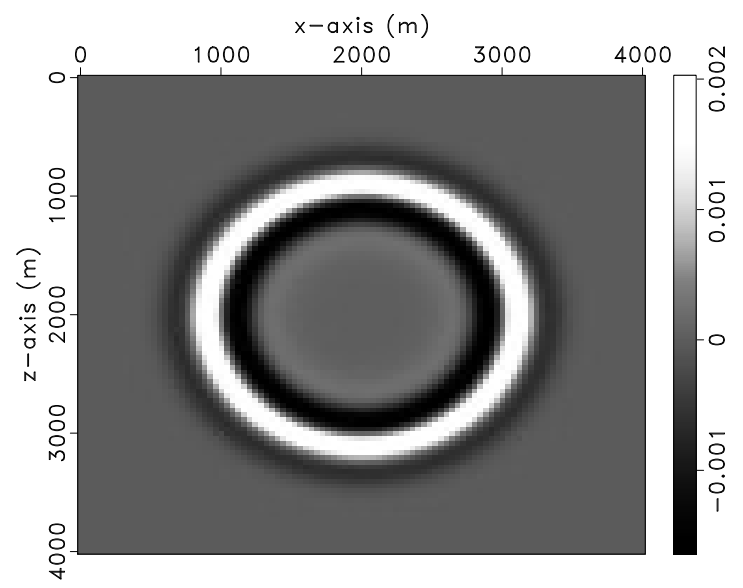

(a)

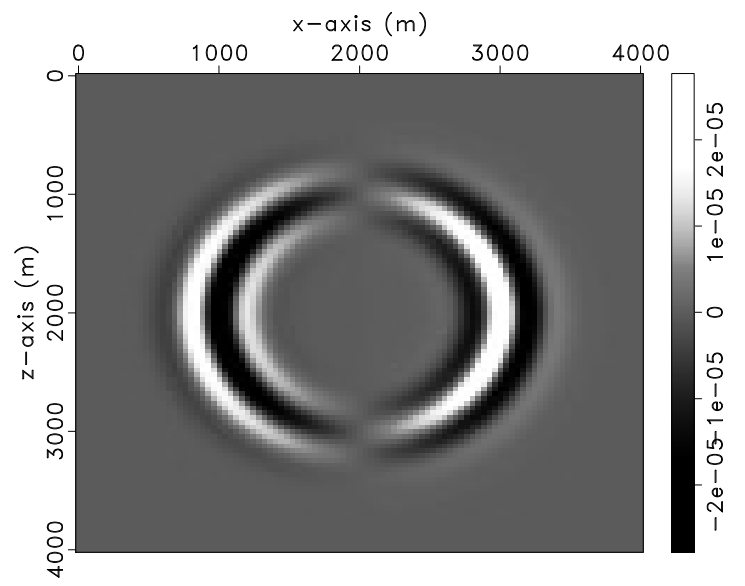

(b)

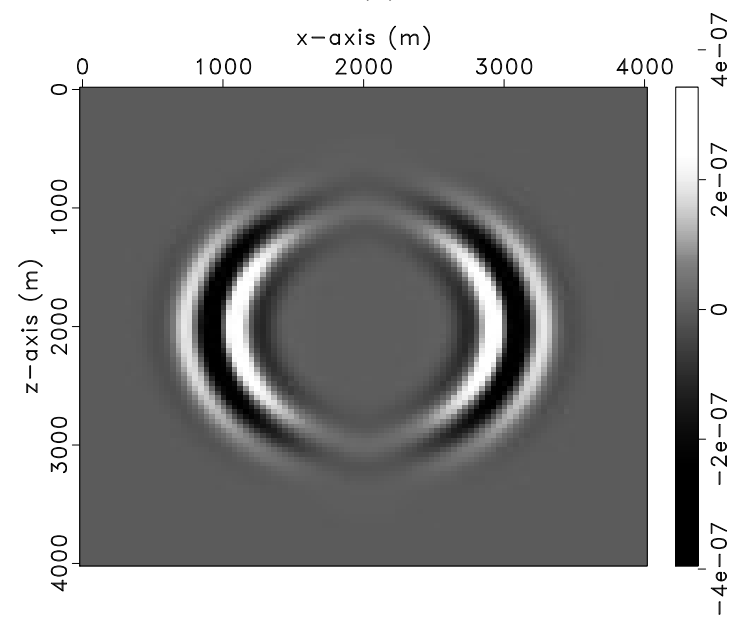

(c)

Figure 4: Snapshot of pressure field $(t=0.748 \mathrm{sec})$ computed using 2-2 finite difference scheme $(h=10 \mathrm{~m}, \Delta t=0.5 \mathrm{~ms})$ and second-order approximation of multipole source $D^{\mathbf{s}} \delta\left(\mathbf{x}-\mathbf{x}^{*}\right):($ a) $\mathbf{s}=(0,0),(\mathrm{b}) \mathbf{s}=(0,1)$, and (c) $\mathbf{s}=(0,2)$. 


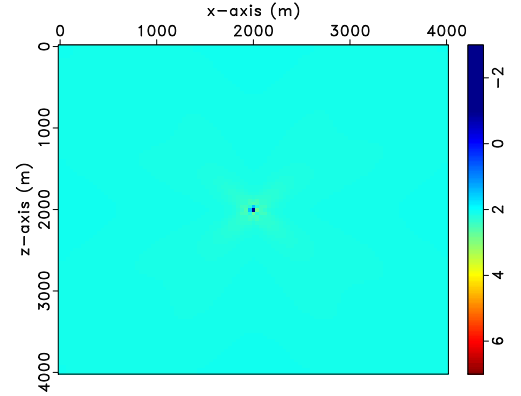

(a)

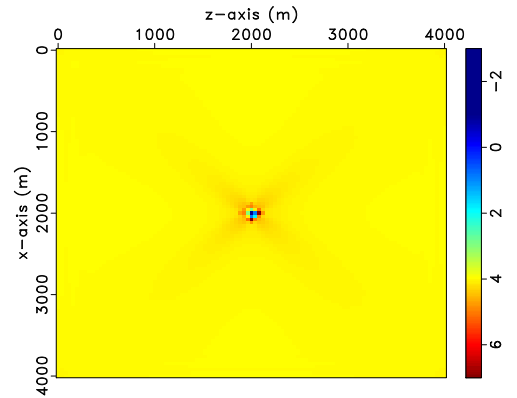

(c)

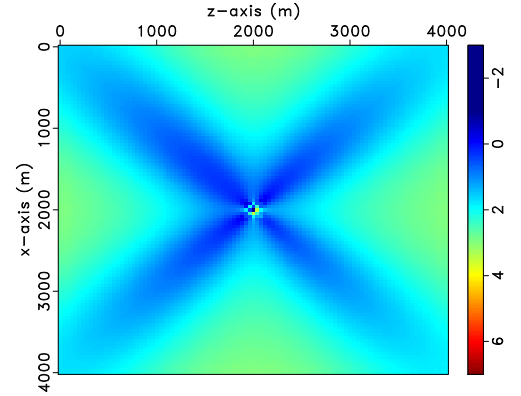

(e)

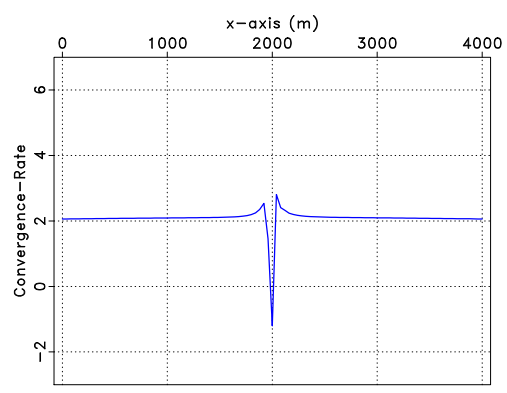

(b)

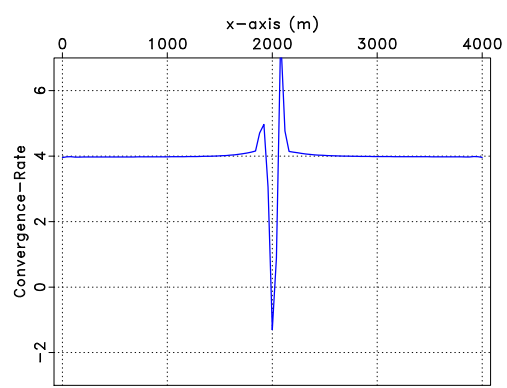

(d)

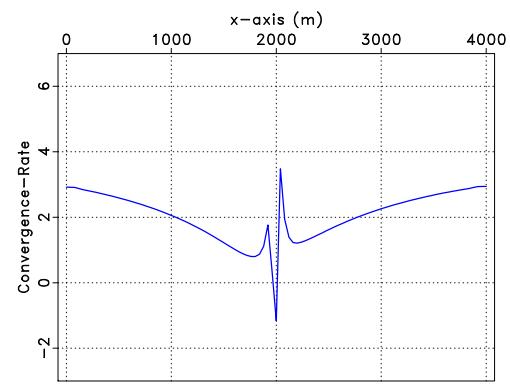

(f)

Figure 5: Convergence rate results for $\mathbf{s}=(0,0)$. Using 2-2 FD scheme and second-order source approximation (a), 2-4 FD scheme and fourth-order source approximation (c), 2-4 FD scheme and second-order source approximation (e). Plots on right column correspond to convergence rates at $z=2000$, related to the left column. 


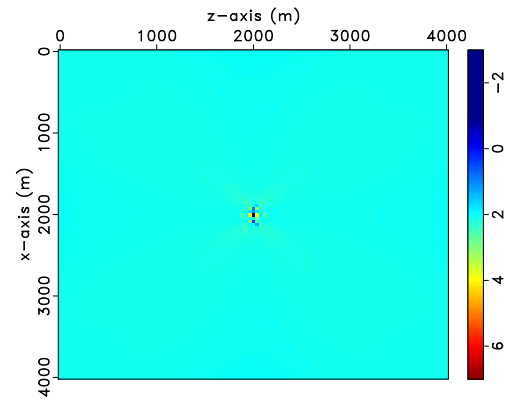

(a)

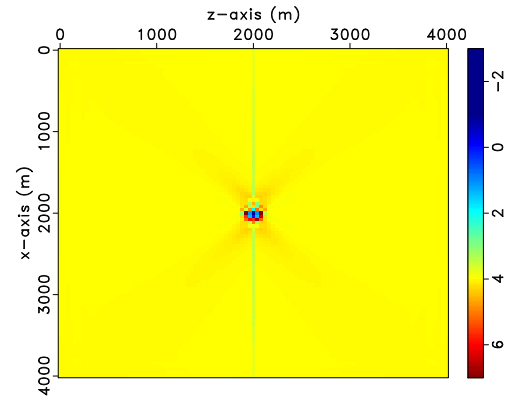

(c)

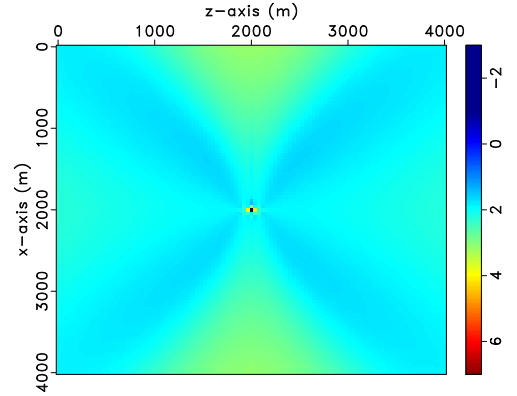

(e)

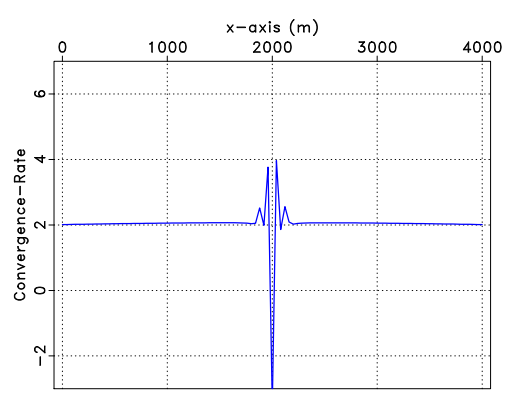

(b)

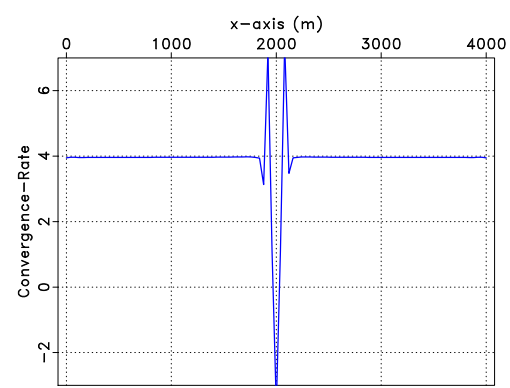

(d)

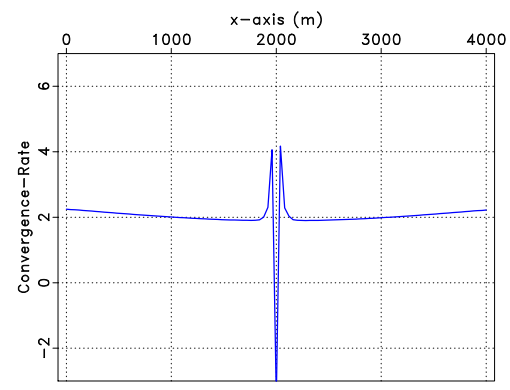

(f)

Figure 6: Convergence rate results for $\mathbf{s}=(0,1)$. Using 2-2 FD scheme and second-order source approximation (a), 2-4 FD scheme and fourth-order source approximation (c), 2-4 FD scheme and second-order source approximation (e). Plots on right column correspond to convergence rates at $z=2000$, related to the left column. 


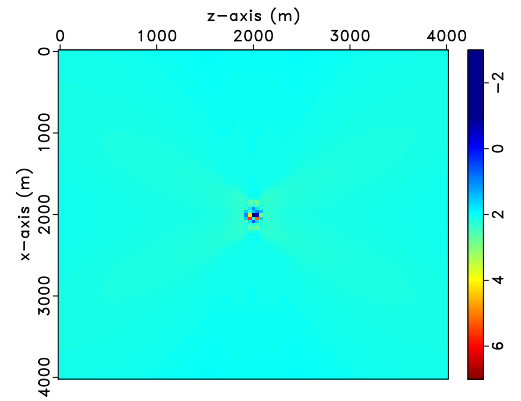

(a)

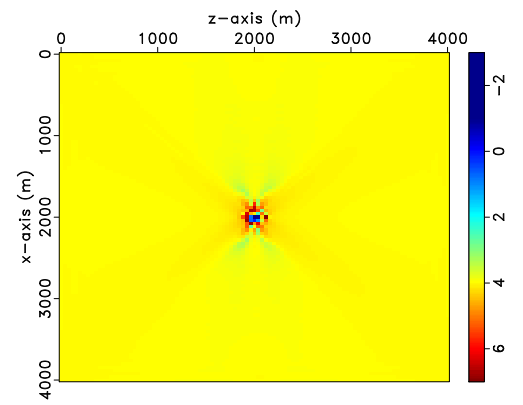

(c)

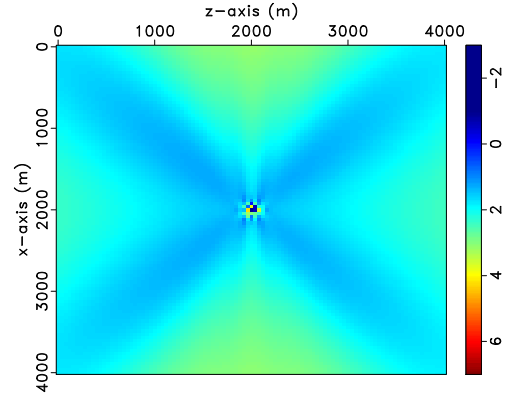

(e)

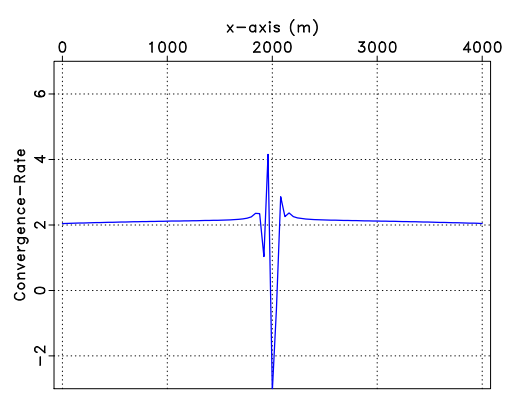

(b)

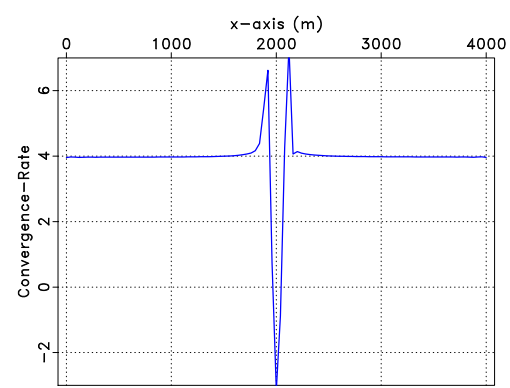

(d)

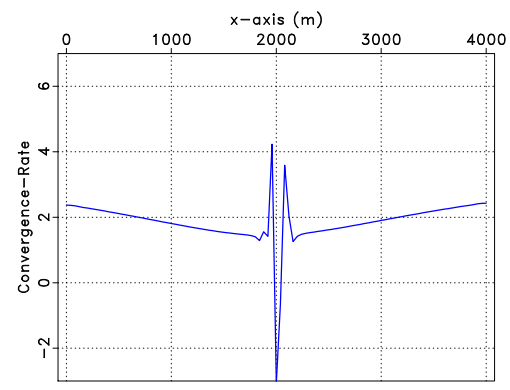

(f)

Figure 7: Convergence rate results for $\mathbf{s}=(0,2)$. Using 2-2 FD scheme and second-order source approximation (a), 2-4 FD scheme and fourth-order source approximation (c), 2-4 FD scheme and second-order source approximation (e). Plots on right column correspond to convergence rates at $z=2000$, related to the left column. 